\title{
The Hadamard Condition for Dirac Fields and Adiabatic States on Robertson-Walker Spacetimes
}

\author{
Stefan Hollands* \\ Department of Mathematics, University of York, \\ York YO10 5DD, UK
}

2 July 2001

\begin{abstract}
We characterise the homogeneous and isotropic gauge invariant and quasifree states for free Dirac quantum fields on Robertson-Walker spacetimes in any even dimension. Using this characterisation, we construct adiabatic vacuum states of order $n$ corresponding to some Cauchy surface. We then show that any two such states (of sufficiently high order) are locally quasi-equivalent. We propose a microlocal version of the Hadamard condition for spinor fields on arbitrary spacetimes, which is shown to entail the usual short distance behaviour of the twopoint function. The polarisation set of these twopoint functions is determined from the Dencker connection of the spinorial Klein-Gordon operator which we show to equal the (pull-back) of the spin connection. Finally it is demonstrated that adiabatic states of infinite order are Hadamard, and that those of order $n$ correspond, in some sense, to a truncated Hadamard series and will therefore allow for a point splitting renormalisation of the expected stress-energy tensor.
\end{abstract}

\section{Introduction}

In many cases of physical interest, for example the early stages of the universe or stellar collapse, one is naturally led to the problem of constructing quantum field theories on a non-static curved spacetime. Numerous papers have been devoted to the study of linear scalar fields on such backgrounds, but less has been done for fields with higher spin, mainly because the analysis of multicomponent fields is technically more involved. The aim of the present paper is to partly fill this gap for the case of a Dirac field on a curved spacetime.

Quantum field theory in curved spacetime (in short, QFT in CST) is best described within the algebraic approach to quantum field theory, which started with the work of Haag and Kastler 11, for an overview see 10. In this approach one deals with a net of $C^{*}$-algebras $\{\mathscr{A}(\mathscr{O})\}_{\mathscr{O} \subset M}$ of observables localised in a spacetime region $\mathscr{O} \subset M$. The algebra $\mathscr{A}=\widehat{\cup_{\mathscr{O} \subset M} \mathscr{A}(\mathscr{O})}$ is called the "quasilocal

*Electronic mail: sh128@york.ac.uk 
algebra'. Quantum states in the algebraic framework are positive normalised linear functionals on the quasilocal algebra. One of the major difficulties of QFT on CST is to pick out physically reasonable states.

It has become widely accepted by now that, for a linear scalar field, the socalled "Hadamard states" are good candidates for physical states. These states are distinguished among other states by the particular form of the singular part of their twopoint function. A mathematically precise definition was given in [18]. The following facts about Hadamard states are known:

They allow for a point-splitting renormalisation of the stress-energy tensor $T_{\mu \nu}$ [34]. Verch [32] has shown that Hadamard states are locally quasi-equivalent and he has also shown local definiteness in the sense of Haag et. al. [12]. Radzikowski 27] discovered that (quasifree) Hadamard states, initially defined by the singular behaviour of the twopoint function in position space, can also be characterised by the so-called 'wave front set' of that twopoint function, a central concept in the mathematical subject called 'microlocal analysis', aimed at describing the singular behaviour of distributions. (Since these techniques do not belong to the daily used toolchest of the theoretical physicist so far, we give a brief introduction to this subject in the appendix.) The microlocal characterisation of Hadamard states is much easier to check in many cases where an explicit expression of the twopoint function cannot be obtained and has already led to important progress in the subject. It played an important rôle in the proof [28] of Kay's conjecture [8] in (axiomatic) QFT in CST, in the derivation of "quantum inequalities" [5] and for the perturbative construction of self-interacting quantum field theories in general globally hyperbolic curved spacetimes [1.

There is a pre-existing notion of a Hadamard state for Dirac fields in a curved spacetime 19 33, analogous to the condition on the singular part of the twopoint function for a linear scalar field. It had been expected that there should also be a microlocal characterisation as for the scalar field, but the details had never been spelled out. One purpose of this paper is to close this gap. The microlocal condition that we propose is similar to that in the spin-0 case. But it differs in that, unless the state in question is assumed to be charge invariant, it needs to be imposed separately for the positive and negative frequency twopoint functions. We show (Thm. IV.2) that our microlocal notion of Hadamard states coincides with the concept based on the short-distance behaviour put forward in [19, 33. Our result is the counterpart of similar theorem by Radzikowski [27] obtained earlier for a scalar field.

New questions also arise in the spin- $1 / 2$ case that have no counterpart in the spin- 0 case. For example, it is natural to ask what the most singular components of the twopoint function are. It appears that the natural mathematical setting to analyse this question is provided by the concept of the 'polarisation set' 3] of a vector valued distribution (such as the twopoint function in the spin- $1 / 2$ case), a notion which refines that of the wave front set of a vector valued distribution. Making use of a theorem by Dencker [3] and the equations of motion, we determine the polarisation set of the twopoint function (Thm. IV.1), corresponding to a Hadamard state of the Dirac field. Along the way, the propagation of singularities for the spinorial Klein-Gordon operator is obtained in Prop. IV.1

On a Robertson-Walker spacetime and for free scalar fields, there exists the 
concept of 'adiabatic states', which was introduced a long time ago by Parker 25] and put on a rigorous mathematical footing by Lüders and Roberts 22 . The main idea behind this concept is the following. If the scale factor $R(t)$ in the Robertson-Walker metric is constant in time, then there is an unambiguous notion of positive frequency solutions to the Klein-Gordon (KG) equation, and one can use these to define a ground state. If $R(t)$ is not constant, then no global ground state exists and the positive freqency solutions have to be determined dynamically off a given Cauchy surface (corresponding to the instant of time at which one wishes to define a vacuum-like state). They are usually found by a WKB-type ansatz and a subsequent iterative approximation process. Adiabatic states of order $n$ (at the time in question) are then the states defined from the positive frequency solutions obtained after $n$ approximation steps. Recently, Junker [15] showed that the problem of finding the 'right' positive frequencies at an instant of time can be viewed as the problem to factorise the KG-operator into positive and negative frequency parts near the Cauchy surface in question.

In this work we present a construction (cf. Def. V.1) of adiabatic states for Dirac fields on $(N+1)$-dimensional Robertson-Walker spacetimes, based on a factorisation of the spinorial KG-operator near a Cauchy surface. While the construction is similar to the scalar case, there are also important differences; for example, special care needs to be taken in order to obtain a manifestly positive state. In Prop. W.1 we explain how the factorisation of the spinorial Klein-Gordon operator (and hence the split into positive and negative frequency solutions) is related to the charge conjugation symmetry of the Dirac equation.

In the scalar case, Lüders and Roberts have shown 22 that the adiabatic states of sufficiently high order are all locally quasi-equivalent and Junker 15 established that those of infinite order are of Hadamard type (which together implies that adiabatic states of sufficiently high order are locally quasiequivalent to a Hadamard state ${ }^{1}$ ). In Sec. IV of this work we show that these results also hold for our adiabatic states in the spin-1/2 case. Furthermore, we show that they correspond, in some sense, to a truncated Hadamard series and therefore allow for a point-splitting renormalisation of the stress tensor $T_{\mu \nu}$ in the same way as scalar fields; in other words, our adiabatic states do not lead to infinite energy fluxes.

Some of our results in the context of Robertson-Walker spacetimes can be generalised to arbitrary globally hyperbolic spacetimes, for example the construction of Hadamard states and a similar criterion for local quasiequivalence, based on the theory of pseudodifferential operators. For these and related issues we refer to a forthcoming paper.

Concerning Thm. IV.1 A proof of this was given in an earlier version of the present paper which was, however, unfortunately incorrect. The first correct proof was given by K. Kratzert [20, see also [21]. These papers in turn built on earlier unpublished work by Radzikowski [29]. We are grateful to K. Kratzert for communicating his results to us and for pointing out the error in an earlier version. In the light of his derivation of that result we were able to repair our earlier proof. It is included here since it is somewhat different from the proof in [20, 21].

\footnotetext{
${ }^{1}$ Originally, it had been claimed in 15 that also adiabatic states of finite ordere were Hadamard. This has been corrected by the author of that paper in the meantime, cf. 16]
} 


\section{The Dirac field on Robertson-Walker space- times}

The aim of this section is to recall the structure of the Dirac equation on Robertson-Walker spacetimes. We assume that the reader has some familiarity with the concept of spinors and the Dirac equation in curved space, as described e.g. in ref. [4].

The homogeneous and isotropic spacetimes in $(N+1)$ dimensions are of the form $M^{\kappa}=\mathbb{R} \times \Sigma^{\kappa}$, the spatial section $\Sigma^{\kappa}$ being the $N$-dimensional sphere $\mathbb{S}^{N}$ for $\kappa=+1$, the Euclidean space $\mathbb{R}^{N}$ for $\kappa=0$ and the (real) hyperbolic space $\mathbb{H}^{N}$ for $\kappa=-1$. The line-element on these spacetimes is

$$
d s_{\kappa}^{2}=d t^{2}-R^{2}(t)\left[d \theta^{2}+f_{\kappa}^{2}(\theta) d \Omega_{N-1}^{2}\right]
$$

where

$$
f_{\kappa}(\theta)= \begin{cases}\sin \theta & \text { for } \kappa=+1 \\ \theta & \text { for } \kappa=0 \\ \sinh \theta & \text { for } \kappa=-1\end{cases}
$$

and $d \Omega_{N-1}^{2}$ is the line-element on $\mathbb{S}^{N-1}$. The above spacetimes are models for a closed, flat or hyperbolic universe with positive, zero or negative curvature. We denote by $n^{\mu} \partial_{\mu}=\partial_{t}$ the future pointing unit vector field normal to the Cauchy surfaces $\Sigma^{\kappa}$ and by $h^{\mu \nu}=g^{\mu \nu}-n^{\mu} n^{\nu}$ the induced (negative definite) metric on $\Sigma^{\kappa}$.

The spaces $\Sigma^{\kappa}$ are homogeneous for the groups $G^{+1}=\operatorname{Spin}(N+1), G^{0}=$ $\operatorname{Spin}(N) \rtimes \mathbb{R}^{N}$ and $G^{-1}=\operatorname{Spin}(N, 1)$ respectively, i.e. $\Sigma^{\kappa}=G^{\kappa} / K$, where $K=\operatorname{Spin}(N)$. We shall omit the superscript $\kappa$ when not necessary and assume that $N$ is odd, $N \geq 3$ in order to simplify the exposition.

In order to bring out the 2 by 2 block matrix form of the Dirac equation in RW-spacetimes, it is useful to define the associated vector bundles (we view $G$ as a $K$ principal fibre bundle over $\Sigma$ )

$$
E^{\tau}=G \times_{\tau} \mathbb{C}^{2^{(N-1) / 2}}, \quad E^{\bar{\tau}}=G \times_{\bar{\tau}} \mathbb{C}^{2^{(N-1) / 2}},
$$

where $\tau$ is the fundamental representation of $K$ and $\bar{\tau}$ the conjugate of that representation. They are related to each other by $Z \tau(k) Z^{-1}=\bar{\tau}(k)$ for all $k \in K$, where $Z$ is some unitary matrix. The spinor and cospinor bundles (restricted to some Cauchy surface $\Sigma(t)=\Sigma \times\{t\}$ ) are related to these bundles by $D M \uparrow \Sigma(t)=E^{\tau} \oplus E^{\tau}$ and $D^{*} M \uparrow \Sigma(t)=E^{\bar{\tau}} \oplus E^{\bar{\tau}}$. Decomposing

$$
C^{\infty}(M, D M) \ni \psi=\left[\begin{array}{l}
\phi \\
\chi
\end{array}\right], \quad \phi, \chi \in C^{\infty}\left(M, E^{\tau}\right),
$$

one can write the Dirac operator as the following 2 by 2 matrix operator:

$$
(i \not \nabla-m) \psi=\gamma^{0}\left(i \partial_{0}+\frac{i N}{2} \partial_{0} \log R-\left[\begin{array}{cc}
m & i R^{-1} \widetilde{\not} \\
i R^{-1} \widetilde{\not} & -m
\end{array}\right]\right)\left[\begin{array}{l}
\phi \\
\chi
\end{array}\right],
$$

where

$$
\gamma^{0}=\left[\begin{array}{cc}
1 & 0 \\
0 & -1
\end{array}\right]
$$


The operator $\widetilde{X}$ is the Dirac operator on $E^{\tau}$, defined without the scale factor $R$. The (generalised) eigenfunctions of this operator (normalised w.r.t. the natural inner product for sections in $\left.E^{\tau}\right), \widetilde{\nabla} \chi_{\vec{k} s}=i s k \chi_{\vec{k} s}$, can be found in terms of special functions. The labels $(\vec{k}, s)$ mean

$\vec{k}=(k, l, m) \quad$ with $\begin{cases}k \in \mathbb{N}+N / 2,0 \leq l \leq k-N / 2, m=0, \cdots, d_{l} & \text { for } \kappa=+1, \\ k \in \mathbb{R}_{+}, l=1,2, \ldots, m=0, \ldots, d_{l} & \text { for } \kappa=0,-1,\end{cases}$

and $s= \pm 1 . d_{l}$ is the degeneracy of the eigenvalue $l+(N-1) / 2$ for the Dirac operator on $\mathbb{S}^{N-1}$, given e.g. in 31. For an explicit representation of the functions $\chi_{\vec{k} s}$ we refer to [2] in the cases when $\kappa=-1,+1$. The case $\kappa=0$ is treated in the appendix.

In order to diagonalise the Dirac Hamiltonian, one defines the spinors

$$
u_{\vec{k} s}^{+}=R^{-N / 2} \mathcal{U}_{k s}\left[\begin{array}{c}
\chi_{\vec{k} s} \\
0
\end{array}\right], \quad u_{\vec{k} s}^{-}=R^{-N / 2} \mathcal{U}_{k s}\left[\begin{array}{c}
0 \\
\chi_{\vec{k} s}
\end{array}\right]
$$

where

$$
\mathcal{U}_{k s}=\frac{1}{\sqrt{2}}\left[\begin{array}{cc}
\sqrt{1+m / \omega_{k}} & -s \sqrt{1-m / \omega_{k}} \\
s \sqrt{1-m / \omega_{k}} & \sqrt{1+m / \omega_{k}}
\end{array}\right]
$$

is a unitary matrix and where $\omega_{k}=\sqrt{m^{2}+k^{2} / R^{2}}$ are the instantaneous frequencies of the mode. The spinor fields $u_{\vec{k} s}^{ \pm}$form a complete set of generalised eigenfunctions for the Hamiltonian

$$
H=-i \not h h^{\mu \nu} \gamma_{\mu} \nabla_{\nu}+\not h m=\left[\begin{array}{cc}
m & i R^{-1} \widetilde{\not} \\
i R^{-1} \widetilde{\not} & -m
\end{array}\right]
$$

and the helicity operator

$$
\Xi=\operatorname{sign}\left(i s^{\mu} \nabla_{\mu}\right)=\operatorname{sign}\left[\begin{array}{cc}
i \widetilde{\not} & 0 \\
0 & i \widetilde{\not}
\end{array}\right], \quad s^{\mu}=|g|^{1 / 2} \epsilon^{\mu \nu \sigma \ldots \rho} n_{\nu} \gamma_{\sigma} \ldots \gamma_{\rho},
$$

with energy $\pm \omega_{k}$ and helicity $s$ at the corresponding instant of time,

$$
H u_{\vec{k} s}^{ \pm}= \pm \omega_{k} u_{\vec{k} s}^{ \pm}, \quad \Xi u_{\vec{k} s}^{ \pm}=s u_{\vec{k} s}^{ \pm} .
$$

We next define the Dirac conjugate and the charge conjugate of a spinor or cospinor, which will be needed later on. Note that, as in any associated vector bundle, there is a one-to-one correspondence between sections $\chi$ in $E^{\tau}$ and smooth $\mathbb{C}^{2^{(N-1) / 2}}$-valued functions $\chi^{\wedge}$ on $G$ such that $\chi^{\wedge}(g k)=\tau(k)^{-1} \chi^{\wedge}(g)$ (a similar identfication can be made for sections in $E^{\bar{\tau}}$ ). The charge conjugate (denoted by $\psi^{c}$ or $C \psi$ ) resp. Dirac conjugate ( denoted by $\bar{\psi}$ or $\beta \psi$ ) of a spinor $\psi$ with decomposition (2) are then defined by

$$
\psi^{c}=\left[\begin{array}{c}
-\left(Z \phi^{\wedge \dagger}\right)^{\vee} \\
\left(Z \chi^{\wedge \dagger}\right)^{\vee}
\end{array}\right], \quad \bar{\psi}=\left[\begin{array}{c}
-\left(\chi^{\wedge \dagger}\right)^{\vee} \\
\left(\phi^{\wedge \dagger}\right)^{\vee}
\end{array}\right] .
$$

$\beta$ is an antilinear map from $D M$ to $D^{*} M$ and $C$ is an antilinear map from $D M$ to $D M$ for which $\{C, H\}=0$. The charge resp. Dirac conjugate of a 
cospinor is defined in a similar way. The action of the symmetry group $G$ of the RW-spacetimes on spinors is defined as follows: Firstly, one has an action $\widetilde{U}$ on sections in $E^{\tau}$, given by ${ }^{2}$

$$
(\widetilde{U}(g) \chi)(\vec{x}):=\left(\chi^{\wedge}(g \cdot)\right)^{\vee}(\vec{x}) .
$$

That group action then extends, by means of the isomorphisms $D M \uparrow \Sigma(t)=$ $E^{\tau} \oplus E^{\tau}$, to an action $U=\widetilde{U} \oplus \widetilde{U}$ on spinors over $M$. An action of $G$ on cospinors is defined by $\bar{U}(g)=\beta U(g) \beta^{-1}$.

An operator $B$ on $C_{0}^{\infty}(M, D M)$ is called isotropic if it commutes with every $U(g), g \in G$. By abuse of notation, we will also use this term for operators defined only on a single Cauchy surface. Such operators have a mode decomposition

$$
\left\langle f_{1}, B f_{2}\right\rangle_{t}=\int \mathrm{d} \vec{k} \sum_{s} \sum_{p q} b_{k s}^{p q} \overline{\tilde{f}_{1 \vec{k} s}^{p}} \tilde{f}_{2 \vec{k} s}^{q}
$$

where $b_{k s}$ is some matrix valued function of the labels and $\tilde{f}_{\vec{k} s}^{ \pm}=\left\langle f, u_{\vec{k} s}^{ \pm}\right\rangle_{t}$, the scalar product on a Cauchy surface $\Sigma(t)$ being defined by

$$
\left\langle f_{1}, f_{2}\right\rangle_{t}=\int_{\Sigma(t)}\left(\bar{f}_{1} \gamma_{\mu} f_{2}\right) \mathrm{d} S^{\mu} .
$$

The correspondence $B \leftrightarrow b$ respects products and taking hermitian adjoints, if these are well-defined. For a proof of the above facts we refer the reader to [17. For later use we mention that $U$ can be viewed as a unitary representation of $G$ on $\mathscr{K}_{t}=L^{2}(\Sigma(t), D M)$, the space of square integrable spinor fields w.r.t. the above inner product. A bounded isotropic operator $B$ on $\mathscr{K}_{t}$ corresponds to an essentially bounded function $b_{k s}$.

The Dirac operator on a globally hyperbolic spacetime (such as RW-spacetimes) has unique retared and advanced fundamental solutions $\$_{R}$ and $\$_{A}$, see [4], satisfying

$$
(i \not \nabla-m) \$_{A}=\$_{A}(i \not \nabla-m)=\mathbb{1}, \quad(i \not \nabla-m) \$_{R}=\$_{R}(i \not \nabla-m)=\mathbb{1},
$$

and (by $J^{ \pm}$we mean the causal future resp. past of a region in spacetime)

$$
\operatorname{supp}\left(\$_{A} f\right) \subset J^{+}(\operatorname{supp}(f)), \quad \operatorname{supp}\left(\$_{R} f\right) \subset J^{-}(\operatorname{supp}(f))
$$

for any compactly supported testspinor $f . \$=\$_{A}-\$_{R}$ is called the causal propagator.

\section{Local algebras for the Dirac field and invari- ant states}

\section{III.1 Local algebras of observables}

The Dirac field on globally hyperbolic manifolds can be quantised in a straightforward manner. For convenience, we review the basic steps here, details can

\footnotetext{
${ }^{2} \widetilde{U}$ is in fact the representation of $G$ induced by the unitary representation $\tau$ of the closed compact subgroup $K$.
} 
be found in 4, which we follow closely. As above, let $\mathscr{K}_{t}=L^{2}(\Sigma(t), D M)$ and $\mathscr{K}_{t}^{\prime}$ its topological dual, identified with $L^{2}\left(\Sigma(t), D^{*} M\right.$ ) (with the inner product defined in a similar way as in (8) $)$. The field algebra $F$ is the uniquely defined unital $C^{*}$-algebra $\operatorname{CAR}\left(\mathscr{K}_{t}\right)$ generated by "time $t$ "-field operators $\Psi_{t}(f)$ and $\bar{\Psi}_{t}(h)$, smeared with square integrable spinor fields $f \in \mathscr{K}_{t}$ resp. cospinor fields $h \in \mathscr{K}_{t}^{\prime}$, which satisfy the "equal time $t$ " anti-commutation relations (CAR's)

$$
\left\{\Psi_{t}(f), \bar{\Psi}_{t}(h)\right\}=\langle\bar{h}, f\rangle_{t} \mathbb{1}, \quad \bar{\Psi}_{t}(f)^{*}=\Psi_{t}(\bar{f}) .
$$

All other anti-commutators are trivial. One also defines $(N+1)$-smeared field operators by $\Psi(f)=\Psi_{t}(\$ f \uparrow \Sigma(t))$, where $f$ is now a compactly supported, smooth spinor field on $M$. In a similar way, one defines $\bar{\Psi}(h)$, where $h \in$ $C_{0}^{\infty}\left(M, D^{*} M\right)$. The field $(N+1)$-smeared field operators satisfy by definition the field equations

$$
\bar{\Psi}((-i \not \nabla-m) h)=\Psi((i \not \nabla-m) f)=0,
$$

and the CAR's (see e.g. ref. 4])

$$
\{\Psi(f), \bar{\Psi}(h)\}=i \not(h, f) \mathbb{1} \quad \text { for all } f \in C_{0}^{\infty}(M, D M), h \in C_{0}^{\infty}\left(M, D^{*} M\right) .
$$

From this it follows at once that the definition of $F$ is in fact independent of the choice of Cauchy surface made above. The algebras of fields localised in a spacetime region $\mathscr{O}$ are defined to be the $C^{*}$-algebras $F(\mathscr{O})$ generated by field operators smeared with test functions supported in $\mathscr{O}$. The algebras of observables localised in $\mathscr{O}$ are given by $\mathscr{A}(\mathscr{O})=F(\mathscr{O})^{\text {even }}$, where we mean the subalgebras generated by products of an even number of fields. From the support properties of the causal propagator, one can easily deduce that spacelike commutativity holds for the algebras of observables,

$$
\left[\mathscr{A}(\mathscr{O}), \mathscr{A}\left(\mathscr{O}^{\prime}\right)\right]=\{0\} \quad \text { if } \mathscr{O} \text { and } \mathscr{O}^{\prime} \text { spacelike. }
$$

The group actions $U$ and $\bar{U}$ of $G$ on spinors resp. cospinors give rise, by standard results on the CAR, to an action by *automorphisms $\alpha_{g}, g \in G$ on the field algebra $F$. The action of these automorphisms on field operators is given by

$$
\alpha_{g} \Psi_{t}(f)=\Psi_{t}(U(g) f), \quad \alpha_{g} \bar{\Psi}_{t}(h)=\bar{\Psi}_{t}(\bar{U}(g) h) .
$$

In the following, we will drop the subscript $t$ at the "time $t$ "-field operators. This should cause no confusion, as it will be clear what is meant from the context.

\section{III.2 Invariant, quasifree states}

A state $\omega$ on $\mathscr{A}=F^{e v e n}$ is said to be isotropic if $\omega(X)=\omega\left(\alpha_{g} X\right)$ for all $g \in G$ and $X \in \mathscr{A}$. It is said to be gauge invariant and quasifree if there exists an operator $0 \leq B \leq \mathbb{1}$ on $L^{2}(\Sigma(t), D M)$ such that

$$
\begin{gathered}
\omega\left(\Psi\left(f_{1}\right) \ldots \Psi\left(f_{n}\right) \bar{\Psi}\left(h_{1}\right) \ldots \bar{\Psi}\left(h_{m}\right)\right)=\delta_{n m} \operatorname{det}\left(\left\langle\bar{h}_{i}, B f_{j}\right\rangle\right)_{i, j=1, \ldots, n}, \\
h_{i} \in L^{2}\left(\Sigma(t), D^{*} M\right), \quad f_{j} \in L^{2}(\Sigma(t), D M) .
\end{gathered}
$$

The term "gauge invariant" refers to the fact that only monomials with the same number of $\Psi$ and $\bar{\Psi}$ fields have a nonzero expectation value in the state 
$\omega$. Clearly, a gauge invariant, quasifree state is isotropic if the corresponding operator $B$ is.

One can easily show that the GNS-construction $\left(\pi_{\omega}, \mathscr{F}_{\omega}, \Omega_{\omega}\right)$ of a gauge invariant quasifree state gives the following: $\mathscr{F}_{\omega}$ is the antisymmetric Fockspace over $\mathscr{K}_{t} \oplus \mathscr{K}_{t}^{\prime}, \Omega_{\omega}$ is the Fock-vacuum and the representation $\pi_{\omega}$ is

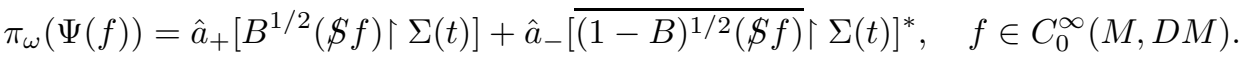

Here, $\hat{a}_{ \pm}$are the destruction operators on $\mathscr{F}_{\omega}$ for particles and antiparticles corresponding to the respective copies of $\mathscr{K}_{t}$, which satisfy the usual anticommutation relations,

$$
\left\{\hat{a}_{+}\left(f_{1}\right)^{*}, \hat{a}_{+}\left(f_{2}\right)\right\}=\left\langle f_{1}, f_{2}\right\rangle_{t}, \quad\left\{\hat{a}_{-}\left(h_{1}\right)^{*}, \hat{a}_{-}\left(h_{2}\right)\right\}=\left\langle h_{1}, h_{2}\right\rangle_{t},
$$

(all other anti-commutators vanish) and $\hat{a}_{+}(f) \Omega_{\omega}=\hat{a}_{-}(h) \Omega_{\omega}=0$.

We next want to ask when two given quasifree, gauge invariant isotropic states are locally quasiequivalent. Let $\omega_{1}$ and $\omega_{2}$ be two such states, corresponding to isotropic operators $B_{1}$ and $B_{2}$ (acting on some Cauchy surface $\Sigma(t))$ with decompositions $b_{1}$ and $b_{2}$ as in Eq. (7).

Theorem III.1. The states $\omega_{1}$ and $\omega_{2}$ are locally quasiequivalent provided

$$
\underset{(k, s)}{\operatorname{ess} \sup }\left[(1+|k|)^{N+\epsilon}\left\|b_{1 k s}-b_{2 k s}\right\|\right]<\infty
$$

(we mean the matrix norm in $\mathbb{C}^{2}$ ) for some $\epsilon>0$.

Proof. Let us choose a region $\mathscr{O}$ of the form $D(\mathscr{C})$, where we mean the domain of dependence of some open subset $\mathscr{C}$ with compact closure of the Cauchy surface $\Sigma(t)$, i.e. the set of all $x \in J^{ \pm}(\mathscr{C})$ such that every past resp. future directed timelike or null curve starting at $x$ hits $\mathscr{C}$. Let us first show that the restrictions of the states to a subalgebra $\mathscr{A}(\mathscr{O}), \mathscr{O}=D(\mathscr{C})$ are quasiequivalent. Regions of this particular shape are convenient, because the algebras $\mathscr{A}(\mathscr{O})$ are then isomorphic to the algebras $\operatorname{CAR}\left(\mathscr{K}_{\mathscr{C}}\right)$ constructed from the Hilbert space $\mathscr{K}_{\mathscr{C}}=L^{2}(\mathscr{C}, D M)$ which is a closed subspace of $\mathscr{K}_{t}$. The restricted states $\omega_{1,2}\left\lceil\mathscr{O}\right.$ then correspond to the operators $E_{\mathscr{C}} B_{1,2} E_{\mathscr{C}}$ on $\mathscr{K}_{\mathscr{C}}$, where $E_{\mathscr{C}}$ denotes the projection on this subspace. One then knows, by a well-known result of Powers and Størmer [26. Thm. 5.1], that the states $\omega_{1}\left\lceil\mathscr{O}\right.$ and $\omega_{2}\lceil\mathscr{O}$ on the algebra $\operatorname{CAR}\left(\mathscr{K}_{\mathscr{C}}\right)$ are quasiequivalent if and only if

$$
\begin{aligned}
\left\|\left(E_{\mathscr{C}} B_{1} E_{\mathscr{C}}\right)^{1 / 2}-\left(E_{\mathscr{C}} B_{2} E_{\mathscr{C}}\right)^{1 / 2}\right\|_{\mathrm{H} . \mathrm{S} .} & <\infty, \\
\left\|\left(E_{\mathscr{C}}\left(I-B_{1}\right) E_{\mathscr{C}}\right)^{1 / 2}-\left(E_{\mathscr{C}}\left(I-B_{2}\right) E_{\mathscr{C}}\right)^{1 / 2}\right\|_{\mathrm{H} . \mathrm{S} .} & <\infty,
\end{aligned}
$$

where we mean the Hilbert-Schmidt norm in $\mathscr{K}_{t}$. Using the Powers-Størmer inequality

$$
\left\|A_{1}-A_{2}\right\|_{\text {H.S. }}^{2} \leq\left\|A_{1}^{2}-A_{2}^{2}\right\|_{\text {tr }},
$$

valid for any two bounded operators (we mean the trace norm), one concludes that Eqs. (10) hold provided

$$
\left\|E_{\mathscr{C}} B_{1} E_{\mathscr{C}}-E_{\mathscr{C}} B_{2} E_{\mathscr{C}}\right\|_{\mathrm{tr}}<\infty .
$$


As above, let $H$ be the Hamilton operator on $\mathscr{K}_{t}$. Trivially, we can write $(p \in \mathbb{R})$

$E_{\mathscr{C}} B_{1} E_{\mathscr{C}}-E_{\mathscr{C}} B_{2} E_{\mathscr{C}}=\left(E_{\mathscr{C}}|H|^{-p / 2}\right)\left[|H|^{p / 2}\left(B_{1}-B_{2}\right)|H|^{p / 2}\right]\left(|H|^{-p / 2} E_{\mathscr{C}}\right)$.

By assumption, the operator $|H|^{p / 2}\left(B_{1}-B_{2}\right)|H|^{p / 2}$ is bounded for $p \leq N+\epsilon$, therefore Eq. (11) will hold if $E_{\mathscr{C}}|H|^{-p / 2}$ can be shown to be Hilbert-Schmidt for such a $p$, since the product of two Hilbert-Schmidt operators is in the trace class. To see this, let us pick an orthonormal basis $\left\{f_{n}\right\}_{n \in \mathbb{N}}$ of spinors in $\mathscr{K}_{\mathscr{C}}$. Then

$$
\begin{aligned}
\left\|E_{\mathscr{C}}|H|^{-p / 2}\right\|_{\text {H.S. }}^{2} & =\sum_{n}\left\langle f_{n}, E_{\mathscr{C}}|H|^{-p} E_{\mathscr{C}} f_{n}\right\rangle_{t} \\
& =\sum_{n} \int \mathrm{d} \vec{k} \sum_{q s}\left(k^{2} / R^{2}+m^{2}\right)^{-p / 2}\left|\tilde{f}_{n \vec{k} s}^{q}\right|^{2} .
\end{aligned}
$$

In order to estimate the r.h.s. of Eq. (12), we exchange the $\mathrm{d} \vec{k}$-integration and the summation over $n$ (this is justified, because the resulting expression turns out to be absolutely convergent). We have,

$$
\sum_{n}\left|\tilde{f}_{n \vec{k} s}^{q}\right|^{2}=\int_{\mathscr{C}} u_{\vec{k} s}^{q}(\vec{x})^{\dagger} u_{\vec{k} s}^{q}(\vec{x}) R^{N} \mathrm{~d}^{N} \vec{x} .
$$

The sum over $q, s, \vec{k}$ at fixed $k$ of the integrand is independent of $\vec{x}$ and equal to twice the spectral function $P_{N}(k)$, defined by

$$
P_{N}(k)=\sum_{l m} \chi_{k l m s}(0)^{\dagger} \chi_{k l m s}(0) .
$$

therefore we have found

$$
\left\|E_{\mathscr{C}}|H|^{-p / 2}\right\|_{\text {H.S. }}^{2}=2 \operatorname{vol}(\mathscr{C}) \int\left(k^{2} / R^{2}+m^{2}\right)^{-p / 2} P_{N}(k) \mathrm{d} k .
$$

The spectral function $P_{N}$ is given by the following expressions

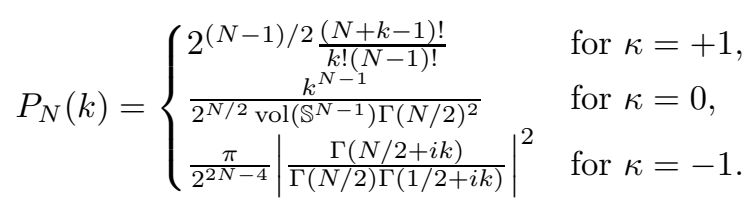

For $\kappa=-1,+1$ a derivation of these may be found in [2, the expression for $\kappa=0$ is derived in the Appendix. $P_{N}(k)$ grows as $k^{N-1}$ for large $k$ for for all the homogeneous spaces $\Sigma=\mathbb{R}^{N}, \mathbb{S}^{N}, \mathbb{H}^{N}$, ensuring that $E_{\mathscr{C}}|H|^{-p / 2}$ is HilbertSchmidt for $p=N+\epsilon$. We have therefore shown that $\omega_{1} \backslash \mathscr{O}$ is quasiequivalent to $\omega_{2}\lceil\mathscr{O}$ for any set $\mathscr{O}$ of the form $D(\mathscr{C})$. Since it is enough to verify local quasiequivalence on a cofinal set of open subsets (such as the set of regions of the type $D(\mathscr{C})$ ), this then proves the theorem.

\section{General properties of Hadamard states for the Dirac field}

The plan of this section is as follows: We first give a microlocal definition of Hadamard states for Dirac quantum fields. After that we determine the 
polarisation set of the twopoint function of such states. Finally, we explain how our microlocal notion of Hadamard states is related to pre-existing notions of Hadamard states for the Dirac fields based on the short-distance behaviour of the twopoint functions. The definitions and results in this section apply to the case of a general, globally hyperbolic spacetime. The reader not familiar with the technical ingredients of the definition will find some notation and results from microlocal analysis in the appendix.

The spatio-temporal twopoint functions of a state $\omega$ are denoted by

$$
\mathbb{G}^{(+)}(h, f):=\omega(\Psi(f) \bar{\Psi}(h)), \quad \mathbb{G}^{(-)}(h, f):=\omega(\bar{\Psi}(h) \Psi(f)),
$$

where $f \in C_{0}^{\infty}(M, D M)$ and $h \in C_{0}^{\infty}\left(M, D^{*} M\right)$. They are assumed to be distributions. We introduce the following (standard) notation: elements in $T_{x}^{*} M$ are denoted by $(x, \xi)$. We write $\left(x_{1}, \xi_{1}\right) \sim\left(x_{2}, \xi_{2}\right)$, if $x_{1}$ and $x_{2}$ can be joined by a null-geodesic $c$ such that $\xi_{1}=\dot{c}(0)$ and $\xi_{2}=\dot{c}(1)$, or if $\left(x_{1}, \xi_{1}\right)=\left(x_{2}, \xi_{2}\right)$ and $\xi_{1}$ null. We write $x_{1} \succ x_{2}$ resp. $x_{1} \prec x_{2}$ if the point $x_{1}$ comes after or before $x_{2}$ according to the parameter on this curve. Moreover, we shall write $\xi \triangleright 0$ if $\xi$ is future-directed and $\xi \triangleleft 0$ if it is past-directed.

Definition IV.1. A quasifree state $\omega$ for the Dirac field is said to be 'Hadamard' if

$$
\begin{aligned}
\mathrm{WF}^{\prime}\left(G^{( \pm)}\right)=\left\{\left(x_{1}, \xi_{1}, x_{2}, \xi_{2}\right)\right. & \in T^{*} M \backslash\{0\} \times T^{*} M \backslash\{0\} \mid \\
& \left.\left(x_{1}, \xi_{1}\right) \sim\left(x_{2}, \xi_{2}\right), \quad \xi_{1} \triangleright(\triangleleft) 0\right\}=: C^{( \pm)}
\end{aligned}
$$

Remark IV.1. It follows from what we say in the proof of Thm. VI.1 that the apparently weaker condition $\mathrm{WF}^{\prime}\left(G^{( \pm)}\right) \subset C^{( \pm)}$already implies equality of these sets.

The conditions on the wave front set of $G^{(+)}$and $G^{(-)}$are in general independent, i.e., there exist states for which $\mathbb{G}^{(+)}$has the desired wave front set but for which $G^{(-)}$has not. However, it is easy to see that this cannot happen for states $\omega$ which are invariant under charge conjugation. By this we mean that $\omega \circ \alpha_{c}=\omega$, where $\alpha_{c}$ is the *-automorphism defined by

$$
\alpha_{c} \Psi(f)=\bar{\Psi}\left(\beta f^{c}\right), \quad \alpha_{c} \bar{\Psi}(h)=-\Psi\left(\beta h^{c}\right) .
$$

Below (Thm. IV.2) we prove that the above condition on the wave front sets of both twopoint functions implies that their singular behaviour in position space is that of a Hadamard fundamental solution. This result will in general not hold if only one twopoint function has the required wave front set.

We now analyse in more detail the microlocal singularity structure of the twopoint functions by applying to them the propagation of singularities theorem Thm. VIII.1 taking $P$ in that theorem to be the spinor Klein-Gordon (KG) operator, given by

$$
P=\square+\frac{1}{4} R+m^{2},
$$

where $R$ is the curvature scalar. This theorem is applicable because

$$
(P \otimes \mathbb{1}) G^{( \pm)}=\left(\mathbb{1} \otimes P^{t}\right) G^{( \pm)}=0,
$$


which is in turn a direct consequence of the Lichnerowicz identity

$$
P=(i \not \nabla-m)(-i \not \nabla-m)
$$

and the fact that $G^{( \pm)}$satisfy the Dirac equation. By ${ }^{t}$ we mean the transpose of an operator acting in the dual bundle (the bundle $D^{*} M$ in the case at hand), obtained from the natural pairing between spinor and cospinor fields on $M$. (The assumption in Thm. VIII.1 that $P$ be of principal type (cf. Def. VIII.3) is fulfilled, because $P$ has a metric principal symbol $p_{0}(x, \xi)=-g^{\mu \nu}(x) \xi_{\mu} \xi_{\nu}$.) In order to apply Thm. VIII.1 we must calculate the Dencker connection $D_{P}$ associated with $P$, defined in Eq. (39). In the case at hand, $D_{P}$ naturally acts in the vector bundle $\pi^{*}\left(D M \otimes \Omega^{1 / 2}\right)\left\lceil\mathcal{Q}_{P}\right.$, where $\Omega^{1 / 2}$ is the line bundle over $M$ of half-densities, $\pi: T^{*} M \rightarrow M$ is the projection map and

$$
\mathcal{Q}_{P}=\left\{(x, \xi) \mid g^{\mu \nu}(x) \xi_{\mu} \xi_{\nu}=0\right\} \subset T^{*} M .
$$

Proposition IV.1. The Dencker connection $D_{P}$ for the operator $P$ is the partial connection in the pull-back of the vector bundle $D M \times \Omega^{1 / 2}$ to $\mathcal{Q}_{P} \subset T^{*} M$ given by

$$
D_{P}=\mathrm{i}_{X_{p_{0}}} \circ \pi^{*}\left(\nabla+\frac{1}{4} d \log |g|\right) .
$$

Here, $X_{p_{0}}$ is the Hamiltonian vector field over $T^{*} M$ corresponding to $p_{0}(x, \xi)$, $\frac{1}{4} d \log |g|$ is the natural connection in $\Omega^{1 / 2}$ and $\mathrm{i}_{X_{p_{0}}}$ is the insertion operator.

Proof. The Dencker connection can be calculated from Eq. (39), taking $\tilde{p}_{0}=\mathbb{1}$ (this means that $q=p_{0}$ in that formula). Introducing an orthonormal frame $e_{\mu}^{a} \mathrm{~d} x^{\mu}$ for the metric tensor, $\eta^{a b}=g^{\mu \nu} e_{\mu}^{a} e_{\nu}^{b}$, and and going to local coordinates, we find

$$
\left\{\tilde{p}_{0}, p_{0}\right\}=0, \quad p^{s}(x, \xi)=2 i S_{\mu}(x) \xi^{\mu}-i e_{\mu}^{a}(x) \partial_{\nu} e_{a}^{\mu}(x) \xi^{\nu}
$$

and

$$
X_{p_{0}}(x, \xi)=-2 \xi^{\mu} \frac{\partial}{\partial x^{\mu}}+2 e_{\sigma}^{a}(x) \partial_{\mu} e_{a \nu}(x) \xi^{\nu} \xi^{\sigma} \frac{\partial}{\partial \xi_{\mu}},
$$

where $S_{\mu}=\frac{1}{8}\left[\gamma^{a}, \gamma^{b}\right] e_{a}^{\nu} \nabla_{\mu} e_{\nu b}$ is the spinor connection. According to Eq. (39), this means that

$$
D_{P}=-2 \xi^{\mu} \frac{\partial}{\partial x^{\mu}}+2 e_{\sigma}^{a}(x) \partial_{\mu} e_{a \nu}(x)-2 S_{\mu}(x) \xi^{\mu}+e_{\mu}^{a}(x) \partial_{\nu} e_{a}^{\mu}(x) \xi^{\nu} .
$$

That this expression agrees with the geometric expression for $D_{P}$ in the statement of this proposition now immediately follows, because $\nabla_{\mu}=\partial_{\mu}+S_{\mu}$, $\frac{1}{4} \partial_{\mu} \log |g|=-\frac{1}{2} e_{\nu}^{a} \partial_{\mu} e_{a}^{\nu}$, and because the differential of the projection map is given by $d \pi\left(\frac{\partial}{\partial x^{\mu}}\right)=\frac{\partial}{\partial x^{\mu}}, d \pi\left(\frac{\partial}{\partial \xi^{\mu}}\right)=0$.

Having derived this tool, we can establish the following result on the polarisation set of the twopoint functions of a Hadamard state.

Theorem IV.1. Any Hadamard state has the following polarisation set:

$$
\begin{array}{r}
\mathrm{WF}_{\text {pol }}^{\prime}\left(G^{( \pm)}\right)=\left\{\left(x_{1}, \xi_{1}, x_{2}, \xi_{2}, w\right) \mid\left(x_{1}, \xi_{1}, x_{2}, \xi_{2}\right) \in C^{( \pm)}, w \in D_{x_{1}} M \otimes D_{x_{2}} M,\right. \\
\text { and } \left.w^{A}{ }_{C^{\prime}} \mathscr{I}\left(x_{1}, x_{2}\right)_{B}{ }^{C^{\prime}}=\lambda \oiint_{1}{ }^{A}{ }_{B} \text { for some } \lambda \in \mathbb{C}\right\} .
\end{array}
$$


Here, unprimed spinor indices refer to the point $x_{1}$, whereas primed ones correspond to $x_{2}$ and $\mathscr{I}\left(x_{1}, x_{2}\right)$ is the bispinor of parallel transport in the bundle $D M$ along a null geodesic joining $x_{1}$ and $x_{2}$. The sets $C^{( \pm)}$had been defined in Eq. (IV.1).

Proof. We aim at using the propagation of singularities theorem, Thm. VIII.1 combined with a deformation argument due to Fulling, Narcowich and Wald [7], first applied in a similar context in [19].

By Thm. VIII.1 and Eq. (14), the polarisation set of $G^{( \pm)}$must be a union of Hamilton orbits corresponding to the operators $P \otimes \mathbb{1}$ and $\mathbb{1} \otimes P^{t}$. By the Prop. IV.1 sections over $\mathcal{Q}_{P}$, annihilated by $D_{P}$ are pull-backs to $T^{*} M$ of sections in $D M$ over null-geodesics which are parallel with respect to $\nabla$. Therefore, two elements $\left(x_{1}, \xi_{1}, x_{2}, \xi_{2}, w\right)$ and $\left(x_{1}^{\prime}, \xi_{1}^{\prime}, x_{2}^{\prime}, \xi_{2}^{\prime}, w^{\prime}\right)$ of $\pi^{*}\left(D M \otimes D^{*} M\right)$ are in the same Hamiltonian orbit if

$$
\begin{array}{r}
\left(x_{1}, \xi_{1}\right) \sim\left(x_{2}, \xi_{2}\right), \quad\left(x_{1}^{\prime}, \xi_{1}^{\prime}\right) \sim\left(x_{2}^{\prime}, \xi_{2}^{\prime}\right), \\
\lambda \cdot w_{B}^{A}=\mathscr{I}\left(x_{1}, x_{1}^{\prime}\right)^{A}{ }_{B^{\prime}} w^{\prime B^{\prime}}{ }_{C^{\prime}} \mathscr{I}\left(x_{2}, x_{2}^{\prime}\right)^{C^{\prime}}{ }_{B},
\end{array}
$$

for some $\lambda \in \mathbb{C}$. Now let $x \in M$ and $\Sigma$ be a Cauchy surface of $M$ through that point. Then there is there is a convex normal $U$ of $x$ and a convex normal neighbourhood $V$ of $\Sigma$, containing $U$, such that there is another spacetime $(\hat{M}, \hat{g})$ with Cauchy surface $\hat{\Sigma}$ and a corresponding causal normal neighbourhood $\hat{V}$ with the properties that: (a) $(V, g)$ is isometric to $(\hat{V}, \hat{g})$ and (b) $\hat{M}$ contains a Cauchy surface $\hat{\Sigma}_{1}$ and a a convex flat neighbourhood $\hat{U}_{1}$ contained in a convex normal neighbourhood $\hat{V}_{1}$ of $\hat{\Sigma}_{1}$ such that $D\left(\hat{U}_{1}\right) \supset \hat{U}$ (we mean the domain of dependence), where $\hat{U}$ corresponds to $U$ under the isometry. By the propagation of singularities theorem, it will be enough to show that $G^{( \pm)} \uparrow U \times U$ has the desired polarisation set, because any pair of null related points can be transported along a null geodesic into a region of that kind. Let $\hat{G}^{( \pm)} \uparrow \hat{V} \times \hat{V}$ be the pull-back of the twopoint functions to the deformed spacetime $(\hat{V}, \hat{g})$. By the propagation of singularities theorem and the equations of motion on the deformed spacetime it will induce a Hadamard distribution on all of $\hat{M}$. Furthermore $\hat{G}^{( \pm)} \uparrow \hat{U} \times \hat{U}$ will have the required polarisation set if $\hat{G}^{( \pm)} \uparrow$ $\hat{U}_{1} \times \hat{U}_{1}$ has, again by the propagation of singularities theorem. But $\hat{U}_{1} \subset \hat{M}$ is contained in a flat portion of spacetime, so effectively our theorem has to be shown for Minkowski space only.

So let $\phi_{\text {mink }}^{( \pm)}$be twopoint functions of a Hadamard state in Minkowski space. By our Thm. IV.2 all Hadamard states differ by a smooth piece only, so we might restrict attention to the vacuum in Minkowski space,

$$
\mathbb{G}_{\mathrm{mink}}^{( \pm)}= \pm(i \not \partial+m) \Lambda_{\mathrm{mink}}^{( \pm)},
$$

where $\Lambda_{\text {mink }}^{( \pm)}$are the ordinary positive resp. negative frequency twopoint functions for the KG-operator $\eta^{\mu \nu} \partial_{\mu} \partial_{\nu}+m^{2}$ in flat space. It is not difficult to see from the definition of the polarisation set and the definition of $G_{\text {mink }}^{( \pm)}$that one must have

$$
\begin{array}{r}
\mathrm{WF}_{\text {pol }}\left(G_{\text {mink }}^{( \pm)}\right) \subset\left\{\left(x_{1}, \xi, x_{2},-\xi, w\right) \mid\left(x_{1}, \xi, x_{2},-\xi\right) \in \mathrm{WF}\left(\Lambda_{\text {mink }}^{( \pm)}\right)\right. \\
\left.w=c \mathbb{1}+\beta \text { for some } c \in \mathbb{C} \text { and } \beta \in \mathbb{C}^{N+1}\right\} .
\end{array}
$$


Now

$$
[(i \not \partial-m) \otimes \mathbb{1}] G_{\text {mink }}^{( \pm)}=G_{\text {mink }}^{( \pm)}[\mathbb{1} \otimes(-i \not \partial-m)]=0,
$$

therefore, using that $\$$ is a principal symbol of $i \not \partial+m$, one can conclude (by the definition of the polarisation set) that

$$
\sharp w=w \notin=0,
$$

where $\left(x_{1}, \xi, x_{2},-\xi\right) \in \mathrm{WF}\left(G_{\text {mink }}^{( \pm)}\right)$. Since the form of $w$ is already restricted by Eq. (15), it is easy to see that these equations imply $\eta^{\mu \nu} \xi_{\mu} \beta_{\nu}=0$ and $c=0$. From the equation $\beta \notin=0$ it follows

$$
\operatorname{tr}\left(\gamma^{\alpha_{1}} \ldots \gamma^{\alpha_{N-1}} \gamma^{N+2} \epsilon_{\alpha_{1} \ldots \alpha_{N-1} \sigma \rho} \beta \not\right)=0
$$

where $\epsilon_{\mu \ldots \nu}$ is the totally antisymmetric tensor in $N+1$ dimensions and

$$
\gamma^{N+2}=\gamma^{0} \ldots \gamma^{d}
$$

Using standard identities for traces of gamma matrices and

$$
\epsilon^{\mu \nu \alpha_{1} \ldots \alpha_{N-1}} \epsilon_{\alpha_{1} \ldots \alpha_{N-1} \sigma \rho}=\delta_{\sigma}^{\mu} \delta_{\rho}^{\nu}-\delta_{\rho}^{\mu} \delta_{\sigma}^{\nu}
$$

we find that $\xi_{\rho} \beta_{\sigma}-\xi_{\sigma} \beta_{\rho}=0$. Since $\xi \neq 0$, this implies $\beta=w=\lambda \cdot \not$, which in Minkowski space is just the condition on the polarisation that was claimed.

In 33, 19, the authors give a definition of the Hadamard condition for Dirac fields in terms of the singular behaviour of the associated twopoint functions in position space. We now investigate the relation between the two definitions. A local version of the definition of [33] 19] may be stated as follows. Let $\mathscr{O}$ be a convex normal neighbourhood in $M$. On $\mathscr{O}$, one defines the bidistributions

$$
H_{n}^{( \pm)}\left(x_{1}, x_{2}\right)=\sum_{j=1}^{(N-1) / 2} U_{j}\left(x_{1}, x_{2}\right) \sigma_{ \pm \epsilon}^{-j}+\sum_{j=0}^{n+1} V_{j}\left(x_{1}, x_{2}\right) \sigma^{j} \log \sigma_{ \pm \epsilon}
$$

where $\sigma$ is the signed squared geodesic distance between the points $x_{1}, x_{2} \in \mathscr{O}$,

$$
\sigma_{\epsilon}\left(x_{1}, x_{2}\right)=\sigma\left(x_{1}, x_{2}\right)+2 i \epsilon\left(t\left(x_{2}\right)-t\left(x_{1}\right)\right)+\epsilon^{2}, \quad \epsilon>0
$$

and $t$ is some global time function. As usual, we mean the bidistribution obtained by smearing with smooth spinor fields first and then taking $\epsilon$ to zero. The bispinors $U_{j}, V_{j}$ are determined recursively by the $N+1$ dimensional analogue of the Hadamard transport equations 23 for the spinorial KG-operator $P$ and depend only the geometry of the spacetime in $\mathscr{O}$. By construction,

$$
(P \otimes \mathbb{1}) H_{n}^{( \pm)}=\left(\mathbb{1} \otimes P^{t}\right) H_{n}^{( \pm)}=0 \quad \bmod C^{n}
$$

on $\mathscr{O}$. The local version of the global Hadamard definition ${ }^{3}$ given in [33, 19] is as follows: A state is said to be locally Hadamard if its associated twopoint function satisfies

$$
G^{( \pm)}= \pm(-i \not \nabla-m) H_{n}^{( \pm)} \text {modulo } C^{n} \text { on } \mathscr{O} \text { for all } n .
$$

\footnotetext{
${ }^{3}$ Not all points in $M$ may be connected by a unique geodesic line, therefore the definition needs to be refined if one also wants to exclude spacelike singularities. We refer to [18] for details.
} 
In fact, it is only necessary to require this equation for ' + ' (as the authors do in ref. 33, 19]), since it automatically follows from this that

$$
\begin{aligned}
G^{(-)} & =i(-i \not \nabla-m) E-\mathbb{G}^{(+)} \\
& =(-i \not \nabla-m)\left(i E-H_{n}^{(+)}\right) \bmod C^{n} \\
& =-(-i \not \nabla-m) H_{n}^{(-)} \bmod C^{n},
\end{aligned}
$$

where we have used that

$$
H_{n}^{(+)}-H_{n}^{(-)}=i E \bmod C^{n+1},
$$

and where $E$ is the causal propagator for $P$. This follows from the fact [6] that $E$ contains the same coefficients $U_{j}, V_{j}$ as $H_{n}^{( \pm)}$, multiplied only by different singular pieces, which, as it is well-known in QFT, may be combined to give the above identity between the different types of propagators.

We now show that Eq. (16) follows from our microlocal definition. This observation has been made first by Radzikowski 27] for the scalar field and his proof can be adjusted to the spinor case as well, although the situation is more complicated.

Theorem IV.2. Let $\omega$ be a Hadamard state in the microlocal sense of Def.IV.1. Then its twopoint functions also satisfy Eq. (16).

Remark IV.2. It can also be shown that the global version of the Hadamard condition (16) (as for example spelled out in [33, 19]) implies the microlocal Hadamard condition. A proof of this is almost identical to the proof of the corresponding statement for the scalar case. We refer to refs. [19, 20, for details.

Proof. Let us denote by $E_{A}, E_{R}, E_{F}, E_{\bar{F}}$ the advanced, retarded, Feynman, antiFeynman parametrices of the spinorial Klein-Gordon operator $P$. They are known to be determined (modulo $C^{\infty}$ ) by the equations

$$
P E_{j}=\mathbb{1} \bmod C^{\infty}, j=A, R, F, \bar{F}
$$

and their wave front sets [13, Thm. 6.5.3]. For the Feynman and anti-Feynman parametrices, these wave front sets read:

$$
\begin{aligned}
\mathrm{WF}^{\prime}\left(E_{F}\right)=\left\{\left(x_{1}, \xi_{1}, x_{2}, \xi_{2}\right) \mid\left(x_{1}, \xi_{1}\right)\right. & \sim\left(x_{2}, \xi_{2}\right), \\
\xi_{1} & \left.\triangleright 0 \text { if } x_{1} \succ x_{2}, \xi_{1} \triangleleft 0 \text { if } x_{1} \prec x_{2}\right\}
\end{aligned}
$$

and

$$
\begin{aligned}
\mathrm{WF}^{\prime}\left(E_{\bar{F}}\right)=\left\{\left(x_{1}, \xi_{1}, x_{2}, \xi_{2}\right) \mid\left(x_{1}, \xi_{1}\right)\right. & \sim\left(x_{2}, \xi_{2}\right), \\
\xi_{1} & \left.\triangleright 0 \text { if } x_{1} \prec x_{2}, \xi_{1} \triangleleft 0 \text { if } x_{1} \succ x_{2}\right\} .
\end{aligned}
$$

Actually, in [13 Thm. 6.5.3] only the case of a scalar operator with metric principal part is treated. Inspection of the proof however shows that it may be extended to operators with metric principal part acting in vector bundles such as $P$. We also need the advanced, retarded, Feynman and anti-Feynman parametrices for the Dirac operator, given by

$$
\$_{j}=(-i \not \nabla-m) E_{j}, \quad j=A, R, F, \bar{F} .
$$


By the anticommutation relations, one infers that

$$
G^{(+)}+G^{(-)}=i \not \$=i\left(\$_{A}-\$_{R}\right)
$$

Let us define

$$
\begin{aligned}
& \mathscr{G}_{F}:=i \mathbb{G}^{(+)}+\Phi_{A}=-i G^{(-)}+\Phi_{R} \\
& G_{\bar{F}}:=-i G^{(+)}+\Phi_{R}=i G^{(-)}+\Phi_{A} .
\end{aligned}
$$

Our aim is now to prove that

$$
G_{F}=\$_{F}, \quad G_{\bar{F}}=\$_{\bar{F}}
$$

modulo a smooth kernel. To this end, we first show that

$$
\mathrm{WF}^{\prime}\left(G_{F}\right) \subset \mathrm{WF}^{\prime}\left(E_{F}\right), \quad \mathrm{WF}^{\prime}\left(\mathbb{G}_{\bar{F}}\right) \subset \mathrm{WF}^{\prime}\left(E_{\bar{F}}\right) .
$$

In order to see why this must be true, consider a point $\left(x_{1}, \xi_{1}, x_{2}, \xi_{2}\right)$ in wave front set of $\mathbb{G}_{F}$ such that $x_{1} \notin J^{-}\left(x_{2}\right)$. Then, because $\$_{A}$ must be zero for such points by the support properties of $E_{A}$, it must hold that $\left(x_{1}, \xi_{1}, x_{2}, \xi_{2}\right) \in$ $\mathrm{WF}^{\prime}\left(G^{(+)}\right)$. Since (by the microlocal Hadamard condition, Eq. (13) $\mathrm{WF}^{\prime}\left(G^{(+)}\right) \subset$ $C^{(+)}$we find that $\left(x_{1}, \xi_{1}, x_{2}, \xi_{2}\right)$ can be in the wave front set of $\mathbb{G}_{F}$ if and only if $\xi_{1} \triangleright 0$. A similar reasoning can be applied for $x_{1} \in J^{-}\left(x_{2}\right)$, this time using the representation $\mathbb{G}_{F}=-i \mathbb{G}^{(-)}+\$_{R}$ and exploiting the microlocal Hadamard condition, Eq. (13), for $G^{(-)}$. Altogether one concludes from this that $\left(x_{1}, \xi_{1}, x_{2}, \xi_{2}\right)$ is in the wave front set of $\boldsymbol{G}_{F}$ if and only if $\left(x_{1}, \xi_{1}\right) \sim\left(x_{2}, \xi_{2}\right)$ and $\xi_{1} \triangleright 0$ for $x_{1} \succ x_{2}$ resp. $\xi_{1} \triangleleft 0$ if $x_{1} \prec x_{2}$, which is just the set Eq. (17). We have therefore shown the first inclusion in Eq. (20). The second inclusion is treated in just the same way.

Now, by definition, we have $\mathbb{G}_{F}+\mathbb{G}_{\bar{F}}=\$_{A}+\$_{R}$. Applying the operator $-i \not \nabla-m$ to the relation [13, II, Eq. 6.6.1]

$$
E_{F}+E_{\bar{F}}=E_{A}+E_{R} \quad \bmod C^{\infty},
$$

we find from this that $\mathbb{G}_{F}-\Phi_{F}=\mathbb{G}_{\bar{F}}-\Phi_{\bar{F}}$ modulo smooth and hence that

$$
\mathrm{WF}^{\prime}\left(G_{F}-\$_{F}\right)=\mathrm{WF}^{\prime}\left(\mathbb{G}_{\bar{F}}-\$_{\bar{F}}\right) \text {. }
$$

We had already shown that $\mathrm{WF}^{\prime}\left(G_{F}\right) \subset \mathrm{WF}^{\prime}\left(E_{F}\right)$ and we also have $\mathrm{WF}^{\prime}\left(\$_{F}\right)=$ $\mathrm{WF}^{\prime}\left((-i \not \nabla-m) E_{F}\right) \subset \mathrm{WF}^{\prime}\left(E_{F}\right)$ (since the wave front set of a distribution cannot become larger when acting upon it with a differential operator), so the set on the left had side of this equation is contained in the set $\mathrm{WF}^{\prime}\left(E_{F}\right)$. By the same arguments the set on the right hand side Eq. (21) must be contained in $\mathrm{WF}^{\prime}\left(E_{\bar{F}}\right)$. It therefore trivially follows that

$$
\mathrm{WF}^{\prime}\left(G_{F}-\$_{F}\right) \subset \Delta_{\mathcal{Q}_{P}}, \quad \mathrm{WF}^{\prime}\left(\mathbb{G}_{\bar{F}}-\$_{\bar{F}}\right) \subset \Delta_{\mathcal{Q}_{P}},
$$

where

$$
\Delta_{\mathcal{Q}_{P}}=\mathrm{WF}^{\prime}\left(E_{F}\right) \cap \mathrm{WF}^{\prime}\left(E_{\bar{F}}\right)=\left\{(x, \xi, x, \xi) \mid g^{\mu \nu}(x) \xi_{\mu} \xi_{\nu}=0\right\} .
$$

Now by definition

$$
(P \otimes \mathbb{1})\left(G_{F}-\$_{F}\right)=\left(\mathbb{1} \otimes P^{t}\right)\left(G_{F}-\$_{F}\right)=0
$$


modulo smooth. We are thus in a position to use the propagation of singularities theorem and we conclude that polarisation sets of the distribution $\mathbb{G}_{F}-\$_{F}$ (and hence the wave front set) must be a union of Hamiltonian orbits for the operator $P$. Using the same arguments as in the proof of the preceding theorem, it is then easy to show that if $(x, \xi, x, \xi)$ is in $\mathrm{WF}^{\prime}\left(\mathbb{G}_{F}-\$_{F}\right)$, then this set must also contain nonzero vectors away from the diagonal, a contradiction. Hence $\mathrm{WF}^{\prime}\left(\mathbb{G}_{F}-\$_{F}\right)=\emptyset$, i.e.,

$$
\mathbb{G}_{F}-\$_{F} \in C^{\infty}
$$

as we wanted to show. Inserting this into Eq. (19) one gets

$$
i G^{(+)}=G_{F}-\$_{A}=(-i \not \nabla-m)\left(E_{F}-E_{A}\right) \bmod C^{\infty} .
$$

It can be extracted from the analysis of the propagators in [6, 9] that

$$
E_{F}-E_{A}=i H_{n}^{(+)} \bmod C^{n+1} .
$$

This holds because all propagators have the same structure as $H_{n}^{( \pm)}$, i.e. the same functions $U_{j}, V_{j}$ multiply different singular parts. These can be combined to give the above equation. Combining this equation with Eq. (22) then proves the theorem.

\section{Adiabatic states}

\section{V.1 Definition of adiabatic states}

For the remainder of this work, we restrict attention to Dirac fields over RWspacetimes. It will be clearly indicated if a result has a wider range of validity.

We begin by constructing adiabatic states for the Dirac fields on RW-spacetimes. By term "adiabatic" we mean that any of these states should give a reasonable mathematical description of the concept of 'empty space' in the very small, i.e. in spacetime regions which are very small compared to the curvature radius. The main ingredient in the construction is a factorisation of the spinorial Klein-Gordon operator into positive and negative frequency parts, which has been considered before in [15], for the spin-0 case. (Such a factorisation is possible on every globally hyperbolic spacetime with a compact Cauchy surface.) Let us outline our construction. Suppose one has constructed a 'Gaußian foliation' $I \ni t \mapsto \Sigma(t)$ of a neighbourhood of a globally hyperbolic spacetime $M$ by smooth Cauchy surfaces $\Sigma(t), I=\left[t_{0}, t_{1}\right]$ denoting some time interval. By 'Gaußian', we mean that the vector field $\partial_{t}=n^{\mu} \partial_{\mu}$ associated to this foliation is geodesic and orthonormal to the Cauchy surfaces, in other words $\nabla_{\nu} n_{\mu}-\nabla_{\mu} n_{\nu}=0$ and $n^{\mu} \nabla_{\mu} n^{\nu}=0$. It is then possible to make the decomposition ( $P$ is the spinorial KG-operator)

$$
P=-\left(i n^{\mu} \nabla_{\mu}+i K+T\right)\left(i n^{\mu} \nabla_{\mu}-T\right) \quad \bmod \mathrm{OP}^{-\infty},
$$

where $T$ is a pseudodifferential operator $(\mathrm{PDO})$ in $\mathrm{OP}^{1}(\Sigma \times I, D M)$ with principal symbol

$$
\sigma_{1}(T)(x, \xi)=\sqrt{-h^{\mu \nu}(x) \xi_{\mu} \xi_{\nu}}
$$


acting 'surface-wise', i.e. arises as a smooth family $\{T(t)\}_{t \in I}$ of operators acting on the surfaces $\Sigma(t)$. Here $K=\nabla_{\mu} n^{\mu}$ is the extrinsic curvature and $H(t)=-i \not h h^{\mu \nu} \gamma_{\mu} \nabla_{\nu}+\not h m$ is the Dirac Hamiltonian. (For the various classes of operators and symbols, we refer the reader to the appendix.) Solutions $T$ to Eq. (23) can be found by inserting the asymptotic expansion for the symbol of the sought-for operator in that equation and then determining the terms in this expansion iteratively. The iterative procedure may be stopped after a finite number $n$ of steps, yielding operators $T_{n}$ which differ from $T$ by an operator of class $\mathrm{OP}^{-n}$. In the case of a RW-spacetime, these will be isotropic. A more detailed discussion of how this works on such a spacetime and how the operators $T$ and $T_{n}$ are defined in that special case is given below, since we do not want to interrupt the present line of argument.

Now fix a $t \in\left[t_{0}, t_{1}\right]$ and define

$$
L_{ \pm}(t)=T(t) \pm H(t),
$$

and similarly operators $L_{n, \pm}(t)$ by taking $T_{n}(t)$ in this formula. Our definition of adiabatic states is based on the following lemma, valid on RW-spacetimes.

Lemma V.1. Let $n \in \mathbb{N} \cup\{\infty\}$. Provided $\dot{R}(t) \neq 0$, then the operators $L_{ \pm}(t)$ can be modified, smoothly in $t$, by a PDO with smooth kernel such that there exist hermitian, isotropic, positive operators $Q(t) \in \mathrm{OP}^{-2}$ on $L^{2}(\Sigma(t), D M)$ satisfying (we omit the reference to the time $t$ )

$$
L_{+} Q L_{+}^{*}+L_{-}^{*} Q L_{-}=\mathbb{1},
$$

In the same way, if $n$ is a natural number there exists $Q_{n}$, positive and isotropic, satisfying this equation for $L_{n, \pm}$.

Note: If $M$ is a spacetime foliated by Cauchy surfaces, then the hermitian adjoint of an operator acting 'surface-wise' (such as in the lemma) is defined w.r.t. to the inner product Eq. (8) on each surface.

Before we prove the lemma, let us give the definition of adiabatic states for the free Dirac field on a RW-spacetime. Let us set

$$
B_{n}=L_{n,+} Q_{n} L_{n,+}^{*}
$$

Then $B_{n} \in \mathrm{OP}^{0}(\Sigma(t), D M)$ is hermitian, isotropic and by the lemma fulfills $0 \leq B_{n} \leq \mathbb{1}$.

Definition V.1. The gauge invariant, quasifree states $\omega_{n}$ defined by the operators $B_{n}$ on $L^{2}(\Sigma(t), D M), n \in \mathbb{N} \cup\{\infty\}$ are called adiabatic states of order $n$ at time $t$.

It is straightforward from the definitions that the twopoint functions of such states are given by (suppressing the subscript $n$ for the moment)

$$
\begin{aligned}
& G^{(+)}(h, f)=\left\langle\left(i n^{\mu} \nabla_{\mu}+T(t)\right) \$ \bar{h}, Q(t)\left(i n^{\mu} \nabla_{\mu}+T(t)\right) \$ f\right\rangle_{t}, \\
& G^{(-)}(h, f)=\left\langle\left(i n^{\mu} \nabla_{\mu}-T(t)^{*}\right) \$ \bar{h}, Q(t)\left(i n^{\mu} \nabla_{\mu}-T(t)^{*}\right) \$ f\right\rangle_{t} .
\end{aligned}
$$


Construction of the operators $T$ and $T_{n}$ in the spin-1/2 case in RWspacetimes: We have,

$$
\begin{aligned}
P & =-(i \not \nabla+m)(i \not \nabla-m) \\
& =-\left(i n^{\mu} \nabla_{\mu}+i K+H\right)\left(i n^{\mu} \nabla_{\mu}-H\right),
\end{aligned}
$$

where we have used that $i n^{\mu} \nabla_{\mu}-H=\not h(i \not \nabla-m)$ and the identities

$$
n^{\mu} \nabla_{\mu} \not h=0, \quad h^{\mu \nu} \gamma_{\mu} \nabla_{\nu} \not h=K,
$$

which follow easily from $n^{\mu} \nabla_{\mu} n^{\nu}=0, \nabla_{\mu} n_{\nu}-\nabla_{\nu} n_{\mu}=0$ and $\nabla_{\mu} \gamma_{\nu}=0$. Therefore Eq. (23) is equivalent to the operator equation

$$
H^{2}+i K H+\left[i n^{\mu} \nabla_{\mu}, H\right]=T^{2}+i K T+\left[i n^{\mu} \nabla_{\mu}, T\right] \bmod \mathrm{OP}^{-\infty}
$$

for all $t \in I$, where $T$ is required to have a principal symbol $\sqrt{-h^{\mu \nu} \xi_{\mu} \xi_{\nu}}$. While the above said holds for any globally hyperbolic spacetime with a Gaußian foliation, we now specialise the discussion to RW-spacetimes, where one of course takes the obvious foliation by the homogeneous surfaces $\Sigma^{\kappa}$. We consider symbols $I \times \mathbb{R} \ni(t, k) \mapsto b_{k s}(t)$, taking values in the complex 2 by 2 matrices and carrying an additional helicity index $s= \pm$. We will often omit reference to the matrix resp. helicity indices and simply write $b \in \mathrm{S}^{n}$ when we mean a matrix valued symbol of class $\mathrm{S}^{n}(I, \mathbb{R}) \otimes M_{2}(\mathbb{C})$. Any such symbol $b$ defines and operator $B$ via Eq. (7). Furthermore, if $b \in \mathrm{S}^{n}$ then $B \in \mathrm{OP}^{n}(\Sigma \times I, D M)$. To see this, one can argue in just the same way as in [15], where a similar statement is proven.

We wish to write $T$ in the form Eq. (7) with some matrix valued symbol $\tau_{k s}$ with principal part equal to $\omega_{k}$. Inserting the ansatz Eq. (7) into Eq. (28), one obtains the following 2 by 2 matrix system of ordinary differential equations with parameter $k$,

$$
i \dot{\tau}+\frac{i N \dot{R}}{2 R} \tau+[\tau, d]+\tau^{2} \stackrel{\bmod \underline{S}^{-\infty}}{=} i \dot{h}+\frac{i N \dot{R}}{2 R} h+[h, d]+h^{2}=: r,
$$

where $h_{k s}=\operatorname{diag}\left[\omega_{k},-\omega_{k}\right]$. To arrive at this equation, we used Eq. (5) and

$$
i n^{\mu} \nabla_{\mu} u^{p}=\sum_{q} i\left[\mathcal{U}^{*}\left(\frac{\partial}{\partial t} \mathcal{U}\right)\right]^{p q} u^{q}=: \sum_{q} d^{p q} u^{q},
$$

where

$$
d_{k s}^{p q}=\left[\begin{array}{cc}
0 & -\frac{i s k m \dot{R}}{2\left(m^{2} R^{2}+k^{2}\right)} \\
\frac{i s k m \dot{R}}{2\left(m^{2} R^{2}+k^{2}\right)} & 0
\end{array}\right] .
$$

The drop-off properties in $k$ required from the the matrix $\tau_{k s}$ uniformly in the time interval $I$, imply that the initial data must be carefully adjusted, and cannot be freely chosen.

We try to find $\tau$ as an asymptotic expansion of its symbol (e.g. in a certain sense 'in powers of $k$ '). First note, that since Eq. (23) should hold only up to the addition of a arbitrary operator of class $\mathrm{OP}^{-\infty}(\Sigma \times I, D M), \tau$ need only be defined up to $\mathrm{S}^{-\infty}$. It is therefore enough to find an asymptotic expansion for $\tau$,

$$
\tau \sim \sum_{j} \vartheta_{j}
$$


where $\vartheta_{j} \in \mathrm{S}^{1-j}$ and matrix valued. In order to get an operator with the right principal symbol we set $\vartheta_{0, s k}(t)=\omega_{k}(t)$ for all $t \in I$. We then define $\vartheta_{j}$ successively in such a way that the $n$ 'th partial sum $\tau_{n}=\sum_{j}^{n} \vartheta_{j}$ in Eq. (31) solves Eq. (29) modulo $\mathrm{S}^{1-n}$. This can be achieved by putting

$$
\vartheta_{n+1}=\frac{-1}{2 \vartheta_{0}}\left(i \dot{\tau}_{n}+\left[\tau_{n}, d\right]+\tau_{n}^{2}+\frac{i N \dot{R}}{2 R} \tau_{n}-r\right) \in \mathrm{S}^{-n}(I, \mathbb{R}) .
$$

Following this procedure we can calculate terms of arbitrary high order in the asymptotic expansion for $\tau$. Any symbol with this expansion will give rise to an operator $T$ factorising the spinorial KG-operator modulo $\mathrm{OP}^{-\infty}$. Moreover, the partial sum $\tau_{n}$ obtained after $n$ iterations will give rise to an isotropic operator $T_{n}$, which will solve Eq. (23) up to $\mathrm{OP}^{-n} . T$ as well as $T_{n}$ have the same principal symbol as $|H|$, i.e. Eq. (24) holds true.

Clearly, if the existence of operators $Q_{n}$ as in the lemma is known for a general globally hyperbolic spacetime, then one can still define operators $B_{n}$ by Eq. (26), and these in turn give quasifree, gauge invariant states $\omega_{n}$.

Proposition V.1. Suppose $(M, g)$ is a spacetime with a compact Cauchy surface $\Sigma$, and suppose there exist operators $Q_{n}(n \in \mathbb{N} \cup\{\infty\})$ as in the above lemma. Then there are operators $0 \leq \tilde{B}_{n} \leq \mathbb{1}$, differing from $B_{n}$ only by an operator of class $\mathrm{OP}^{-n-1}$, such that $C \tilde{B}_{n} C^{-1}=\mathbb{1}-\tilde{B}_{n}$ for all $n$.

Remark V.1. It follows from the definition of the charge conjugation automorphism that the states $\tilde{\omega}_{n}$ corresponding to $\tilde{B}_{n}$ are charge invariant.

Proof. We treat the case of infinite order first and suppress the subscript $n$. We also introduce the notation $A^{c}=C A C^{-1}$ for operators $A$ acting on spinors. We need to show that $B$ can be modified modulo $C^{\infty}$ to an operator $\tilde{B}$ such that $\tilde{B}^{c}=\mathbb{1}-\tilde{B}$ and $0 \leq \tilde{B} \leq \mathbb{1}$. Taking the transpose of Eq. (23) we obtain

$$
P^{t}=-\left(-i n^{\mu} \nabla_{\mu}-i K-T^{t}\right)\left(-i n^{\mu} \nabla_{\mu}+T^{t}\right) \bmod \mathrm{OP}^{-\infty} .
$$

It is not difficult to see that $\overline{P f}=P^{t} \bar{f}$ and $T^{t} \bar{f}=\overline{T^{*} f}$. From this it follows that

$$
P=-\left(i n^{\mu} \nabla_{\mu}+i K-T^{*}\right)\left(i n^{\mu} \nabla_{\mu}+T^{*}\right) \quad \bmod \mathrm{OP}^{-\infty}
$$

But multiplying Eq. (23) with $C$ from both sides and using that $\left(i n^{\mu} \nabla_{\mu}\right)^{c}=$ $-i n^{\mu} \nabla_{\mu}$, we also have that

$$
P=-\left(i n^{\mu} \nabla_{\mu}+i K-T^{c}\right)\left(i n^{\mu} \nabla_{\mu}+T^{c}\right) \quad \bmod \mathrm{OP}^{-\infty} .
$$

Therefore, since the principal symbols of $T^{*}$ and $T^{c}$ are equal and since the factorisation is unique modulo $\mathrm{OP}^{-\infty}$ once this information is known, we have shown that

$$
T^{*}=T^{c} \bmod \mathrm{OP}^{-\infty} .
$$

We redefine $T$ by $\frac{1}{2}\left(T+T^{* c}\right)$ and $Q$ by $\frac{1}{2}\left(Q+Q^{c}\right)$ and $L_{ \pm}$by inserting the modified definition of $T$. These redefined operators will then satisfy (remembering 
that that $H^{c}=-H$ and using that $A^{c *}=A^{* c}$ for any operator $A$ acting on spinor fields over $\Sigma(t)^{4}$.)

$$
Q^{c}=Q, \quad Q \geq 0, \quad L_{ \pm}^{c}=L_{\mp}^{*}
$$

and

$$
L_{+} Q L_{+}^{*}+L_{-}^{*} Q L_{-}=\mathbb{1}+A,
$$

where $A \in \mathrm{OP}^{-\infty}$. Since the left hand side of this equation is invariant under charge and hermitian conjugation and positive, we find $A^{c}=A, A^{*}=A$ and $\mathbb{1}+A \geq 0$. Since $\Sigma(t)$ is compact, $A$ is a compact operator and the projectors on all nonzero eigenspaces have a smooth kernel. Let $F$ be the projector on the (finite dimensional) kernel of $\mathbb{1}+A$. Then $R=\mathbb{1}+A+F$ is strictly positive, $[F, R]=0, F R=F, F^{c}=F, R^{c}=R$. Let us write

$$
\tilde{L}_{ \pm}=R^{-1 / 2} L_{ \pm} R^{-1 / 2}, \quad \tilde{Q}=R^{1 / 2} Q R^{1 / 2} .
$$

Then $\tilde{L}_{ \pm}^{c}=\tilde{L}_{\mp}^{*}, \tilde{Q} \geq 0$, and the operator $\tilde{B}$ defined by

$$
\tilde{B}=\tilde{L}_{+} \tilde{Q} \tilde{L}_{+}^{*}+\frac{1}{2} F
$$

satisfies $B=\tilde{B}$ modulo smooth, $\tilde{B}^{c}=\mathbb{1}-\tilde{B}$ and $0 \leq \tilde{B} \leq \mathbb{1}$, providing us thus with a modified operator with the desired properties. For arbitrary $n \geq 1$, one proceeds in a similar way, this time using that $T_{n}^{*}=T_{n}^{c}$ modulo $\mathrm{OP}^{-n-1}$.

Proof of lemma V.1

Proof. The argument establishing the existence of $Q_{n}$ as in the lemma is the same for all $n \geq 1$, therefore, to lighten the notation we will only treat the case $n=\infty$ and drop the reference to $n$. We set $\ell_{ \pm}=\tau \pm h$. The $\ell_{ \pm}$are then related to the PDO's $L_{ \pm}$in the statement of the lemma by Eq. (7). One finds after the first iteration,

$$
\ell_{+, k s}=2\left[\begin{array}{cc}
\omega & 0 \\
0 & -\frac{i \partial_{t}\left(R^{N / 2} \omega_{k}\right)}{R^{N / 2} \omega_{k}}
\end{array}\right], \quad \ell_{-, k s}=2\left[\begin{array}{cc}
0 & 0 \\
0 & \omega_{k}-\frac{i \partial_{t}\left(R^{N / 2} \omega_{k}\right)}{R^{N / 2} \omega_{k}}
\end{array}\right]
$$

modulo $\mathrm{S}^{-1}$. Further iterations change $\tau$ only by symbols of order less or equal -1 and will therefore not affect the above form of $\ell_{ \pm}$. Only the above form of the $\ell_{ \pm}$is used to argue the existence of $Q$, therefore the adiabatic order $n$ is not important for our argument, as long as $n \geq 1$. The proof of the lemma then amounts to show that $\tau$ can be modified by a symbol of class $\mathrm{S}^{-\infty}$ such that one can find a $q \in \mathrm{S}^{-2}$ (or rather one for each helicity), taking values in the complex 2 by 2 matrices, such that $\left({ }^{*}\right.$ denotes the hermitian adjoint of a matrix and $\mathbb{1}$ the identity matrix)

$$
\ell_{+, k s} q_{k s} \ell_{+, k s}^{*}+\ell_{-, k s}^{*} q_{k s} \ell_{-, k s}=\mathbb{1}, \quad q_{k s}=q_{k s}^{*}, \quad q_{k s} \geq 0 \quad \forall k \geq 0, s= \pm .
$$

The PDO $Q$ corresponding to $q$ by Eq. (7) then obviously fulfills the claim of the lemma (note that the integral/sum in Eq. (7) is over positive $k$ only). Taking

\footnotetext{
${ }^{4}$ Here one must use that the foliation is Gaußian.
} 
the matrix adjoint of Eq. 333, one observes that $q$ can be taken to be hermitian. One may regard Eq. (33) as a linear equation for $q$ at each value of $k$ and the helicity index $s$, and thus write it as a 4 by 4 matrix system for the matrix entries of $q$

$$
M_{k s}\left[\begin{array}{l}
q_{k s}^{11} \\
q_{k s}^{22} \\
q_{k s}^{12} \\
q_{k s}^{21}
\end{array}\right]=\left[\begin{array}{l}
1 \\
1 \\
0 \\
0
\end{array}\right],
$$

where $M_{k s}$ is a 4 by 4 matrix determined from the entries of $\ell_{ \pm, k s}$. Only using the above from of $\ell_{ \pm}$and the fact that $S^{m} S^{n} \subset S^{n+m}$ one finds from Eq. (33)

$$
\begin{aligned}
M & =D(\mathbb{1}+A) \quad \text { where } \\
D_{k s} & =4 \operatorname{diag}\left[\omega_{k}^{2}, \omega_{k}^{2},-i R^{-N / 2} \partial_{t}\left(R^{N / 2} \omega_{k}\right), i R^{-N / 2} \partial_{t}\left(R^{N / 2} \omega_{k}\right)\right], \quad A \in \mathrm{S}^{-1} .
\end{aligned}
$$

Hence, if $\dot{R}(t) \neq 0$, then $M_{k s}$ has a matrix inverse in $\mathrm{S}^{-2}$ for large $k$,

$$
M_{k s}^{-1}=D_{k s}^{-1} \sum_{m=0}^{\infty} A_{k s}^{m}
$$

Eq. (34) may therefore be inverted for large $k$ and gives us a solution $q$ to Eq. (33). It follows directly from the above form of $M^{-1}$ that $q_{k s}$ has $\operatorname{diag}\left[\left(2 \omega_{k}\right)^{-2},\left(2 \omega_{k}\right)^{-2}\right]$ as a principal symbol, therefore $q_{k s}$ is positive definite for large $k$. We have thus constructed a solution to Eq. (33) if $k$ is greater than some $k_{0} \geq 0$. To find such a $q$ also for $0 \leq k \leq k_{0}$, we may redefine

$$
\tau(t, k)= \begin{cases}\tau\left(t, k_{0}\right) & \text { for } 0 \leq k \leq k_{0}, \\ \tau(t, k) & \text { for } \quad k \geq k_{0},\end{cases}
$$

and arbitrarily for $k \leq 0$, since $T$ by definition does not depend on $\tau(t, k)$ for $k \leq 0$. By what we have already shown, such a $\tau$ trivially allows for a hermitian, positive solution of Eq. (33), but it is not yet a symbol (because its dependence on $k$ is not smooth). We might however change the above definition of $\tau$ in an arbitrary small neighbourhood of $k_{0}$ to make it smooth (and hence a symbol), without making the corresponding matrix $M$ singular. As we mentioned earlier, the resulting matrix $q$ is automatically hermitian. By easy arguments based on the continuity of the construction, it will also remain positive, if that change is made arbitrarily small.

\section{Properties of adiabatic states}

Proposition VI.1. The states $\omega_{n}$ are locally quasiequivalent to a Hadmard state if $n \geq N$. Furthermore, the difference between the twopoint functions $\mathbb{G}_{n}$ of $\omega_{n}$ and those of a Hadamard state is given by a $C^{n-N+1}$ kernel.

Proof. According to Thm.VI.1 an adiabatic state of infinite order (defined, as described above, by a symbol $\left.b \in \mathrm{S}^{0}(I, \mathbb{R})\right)$ is Hadamard, so it is sufficient to show that adiabatic states of order $n$ (described by a symbol $b \in \mathrm{S}^{0}(I, \mathbb{R})$ ) are 
locally quasiequivalent to such a state. Now $b-b_{n}$ is by definition a symbol of order $-(n+1)$, so in particular,

$$
\left\|b_{k s}-b_{n k s}\right\| \leq c|1+k|^{-n-1} \quad \text { for all } k \geq 0 .
$$

The criterion on local quasiequivalence, Thm. III.1 then immediately proves that the states are locally quasiequivalent if $n \geq N$.

Let $A_{n} \in \mathrm{OP}^{-n-1}(\Sigma(t), D M)$ be the operator associated to the symbol $a_{n}=b-b_{n}$ via Eq. (7) at some $t \in I$. By standard theorems, e.g. in [30. II, Prop. 2.7], the associated kernel on $\Sigma(t) \times \Sigma(t)$ is in $C^{n-N+1}$ for $n \geq N-1$. The difference of the twopoint function of an adiabatic state of infinite order and one of order $n$ is

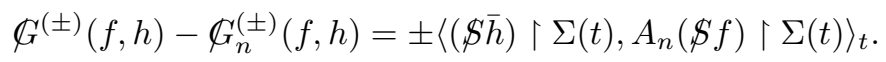

Now the causal propagator $\$$ propagates $m$ times differentiable initial data to $m$ times differentiable solutions. Therefore the above difference must $(n-N+1)$ times differentiable in $M \times M$.

Theorem VI.1. The adiabatic states are Hadamard in the sense of Def. IV.1.

Proof. In order to prove that $G^{( \pm)}$has the wave front set described in Def. IV.1. we shall employ the following result due to W. Junker [15, Thm 3.12]. This result has originally been obtained for scalar fields, but a careful analysis of the proof shows that it can be adapted to the spinor case. We present here a modified version which is tailored to our situation. $E$ is the causal propagator for the spinorial Klein-Gordon operator $P$.

Theorem VI.2. Let $Q(t)$ be an elliptic PDO on $\Sigma(t)$. Let $I$ be an interval containing $t$ and $A_{ \pm} \in \mathrm{OP}(\Sigma \times I, D M)$ such that there exist PDO's $R_{ \pm} \in$ $\mathrm{OP}(\Sigma \times I, D M)$ which have the property $R_{ \pm}\left(i n^{\mu} \nabla_{\mu}+A_{ \pm}\right)=P$ modulo smooth and

$$
\mathcal{Q}_{R_{ \pm}} \subset\left\{(x, \xi) \in T^{*} M \backslash\{0\} \mid \xi \triangleright(\triangleleft) 0\right\},
$$

where $\mathcal{Q}_{R_{ \pm}}$is defined in Eq. (38). Then the spinorial bidistributions

$$
\Lambda^{( \pm)}(h, f)=\left\langle\left(i n^{\mu} \nabla_{\mu}+A_{ \pm}(t)\right) E \bar{h}, Q(t)\left(i n^{\mu} \nabla_{\mu}+A_{ \pm}(t)\right) E f\right\rangle_{t}
$$

have wave front set $\mathrm{WF}^{\prime}\left(\Lambda^{( \pm)}\right) \subset C^{( \pm)}$.

We apply the lemma to $A_{-}=-T$ and $A_{+}=T^{*}$ and $Q$ as in the definition of the twopoint functions, Eq. (27). Then Eq. (23), and Eq. (28) provide us with operators

$$
R_{-}=-\left(i n^{\mu} \nabla_{\mu}+i K+T\right), \quad R_{+}=-\left(i n^{\mu} \nabla_{\mu}+i K-T^{*}\right)
$$

as in the statement of the above theorem. Clearly, since $\sigma_{1}(T)=\sigma_{1}\left(T^{*}\right)=$ $\sqrt{-h^{\mu \nu} \xi_{\mu} \xi_{\nu}}$

$$
\mathcal{Q}_{R_{ \pm}}=\left\{(x, \xi) \mid n^{\mu} \xi_{\mu}= \pm \sqrt{-h^{\mu \nu} \xi_{\mu} \xi_{\nu}}\right\}=\{(x, \xi) \mid \xi \triangleright(\triangleleft) 0\}
$$

Noting that $(-i \not \nabla-m) E=\$$ and using the fact that the wave front set cannot become larger upon acting with a PDO on a distribution, we can apply Junker's 
theorem to $G^{( \pm)}$(given by Eq. (27) ) and obtain $\mathrm{WF}\left(G^{( \pm)}\right) \subset C^{( \pm)}$. It remains to show equality in the above inclusions. The anticommutation relations imply that $G^{(+)}+G^{(-)}=i \$$. If the causal propagator $\$$ had wave front set $W=$ $\left\{\left(x_{1}, \xi_{1}, x_{2}, \xi_{2}\right) \mid\left(x_{1}, \xi_{1}\right) \sim\left(x_{2}, \xi_{2}\right)\right\}=C^{(+)} \cup C^{(-)}$(and this will indeed be shown) then

$$
W=\mathrm{WF}^{\prime}(\$) \subset \mathrm{WF}^{\prime}\left(\mathbb{G}^{(+)}\right) \cup \mathrm{WF}^{\prime}\left(G^{(-)}\right) \subset C^{(+)} \cup C^{(-)}=W,
$$

thus in fact equality would hold in the above inclusions. We have to show that $\$$ has indeed wave front set $W$. From $\$^{c}=\$$ and the antilinearity of the charge conjugation it follows that

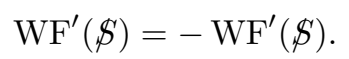

One has (see [4]), $\$ \uparrow \Sigma \times \Sigma=i \not \iota \mathbb{1}$ where $\mathbb{1}$ means the identity on the Cauchy surface. From [15, Thm. 2.22], one knows that

$$
\begin{aligned}
& \mathrm{WF}^{\prime}(\not \uparrow \Sigma \times \Sigma) \subset d \phi^{t} \circ \mathrm{WF}^{\prime}(\not):=\left\{\left(x_{1}, d \phi_{x_{1}}^{t}\left(\xi_{1}\right), x_{2}, d \phi_{x_{2}}^{t}\left(\xi_{2}\right)\right) \mid\right. \\
& \left.\left(\phi\left(x_{1}\right), \xi_{1}, \phi\left(x_{2}\right), \xi_{2}\right) \in \mathrm{WF}^{\prime}(\not), \quad x_{1}, x_{2} \in \Sigma\right\}
\end{aligned}
$$

where $\phi: \Sigma \rightarrow M$ is the embedding map. Now assume that $\left(x_{1}, \xi_{1}, x_{2}, \xi_{2}\right)$ is in $W$ but not in the wave front set of $\$$. By the propagation of singularities, we can assume that there is an element $(x, \xi, x, \xi), x \in \Sigma$, which is in $W$ but not in $\mathrm{WF}^{\prime}(\$)$. By Eq. (35), also $(x,-\xi, x,-\xi) \notin \mathrm{WF}^{\prime}(\$)$. Since $\xi$ must be a nonzero null covector, it is impossible that the nonzero element $\left(x, d \phi_{x}^{t}(\xi), x, d \phi_{x}^{t}(\xi)\right)$ is in $d \phi^{t} \circ \mathrm{WF}^{\prime}(\$) \supset \mathrm{WF}^{\prime}($ in $1 \mathbf{1})$. But the latter set is actually equal to

$$
\mathrm{WF}^{\prime}\left(\delta^{(N)}\right)=\left\{(x, \xi, x, \xi) \mid \xi \in T^{*} \Sigma \backslash\{0\}\right\},
$$

and so must contain any element of that form, a contradiction.

\section{Comments}

1. At adiabatic order zero we get $B_{0}(t)=\frac{1}{2}|H(t)|^{-1}(H(t)+|H(t)|)$. i.e. $B_{0}(t)$ projects on the instantaneous positive frequency solutions at time $t$. Clearly, if $R$ is constant in a neighbourhood of $t$, then there are no further corrections to this operator at higher adiabatic orders, and $B_{0}(t)$ defines a pure quasifree Hadamard state. If $R$ is not constant near $t$, then the operators $B_{n}(t)$ give states which are not Hadamard in general but only reproduce the highest order singularities of the Hadamard form, in the sense that ${ }^{5}$ (modulo $C^{n-N+1}$ ),

$$
\begin{aligned}
& G_{n}^{( \pm)}\left(x_{1}, x_{2}\right)= \\
& \quad \pm(i \not \nabla+m)\left[\sum_{j=1}^{(N-1) / 2} U_{j}\left(x_{1}, x_{2}\right) \sigma_{ \pm \epsilon}^{-j}+\sum_{j=0}^{n-N+2} V_{j}\left(x_{1}, x_{2}\right) \sigma^{j} \log \sigma_{ \pm \epsilon}\right]
\end{aligned}
$$

Hence, for $n \geq N$, adiabatic states will allow for a point-splitting renormalisation of the stress-energy tensor $T_{\mu \nu}$ as described e.g. in [34] (the

\footnotetext{
${ }^{5}$ Note that the next term in this series would be $\sigma^{n-N+3} \log \sigma$, which is $(n-N+2)$ times differentiable.
} 
difference to a Hadamard state must be at least in $C^{1}$, because the stress tensor contains 1 derivative), but such of lower order will not in general. In other words, we see that any adiabatic vacuum state which allows for a point splitting renormalisation of the stress-energy tensor will be locally quasiequivalent to a Hadamard state.

2. In 24, the authors observe that the expected stress tensor of a Dirac field diverges for the 'energy-minimising states' proposed in that work. This is explained by our analysis, since their states are simply the adiabatic states of order zero just discussed in diguise.

3. Following the strategy of Lüders and Roberts [22] for the scalar KleinGordon field, 35] proposes another definition of adiabatic states for the Dirac field. We do have some doubts as to whether their definition really yields a positive state, moreover the rôle of positive and negative frequency modes remains obscure in [35]. It is therefore difficult to see how their definition relates to ours. In view of the analysis carried out in this paper, we do not believe that the states proposed in [35] are of Hadamard type, as suggested by the author.

4. It is possible to construct a Hadamard state in a general globally hyperbolic spacetimes along the same lines as in the previous section if one can construct a hermitian, positive $\mathrm{PDO} Q$ such that

$$
L_{+} Q L_{+}^{*}+L_{-}^{*} Q L_{-}=\mathbf{1} .
$$

However, unlike in the simple case of a RW-spacetime, the construction of such a $Q$ seems to be harder. We are currently working on this problem.

\section{Appendix}

\section{VIII.1 Spinors on flat space and representation theory}

In this appendix we find the (generalised) eigenfunctions of the spatial Dirac operator $\widetilde{X}$ on $\mathbb{R}^{N}$. To this end, we first write this operator in polar coordinates,

$$
\widetilde{\nabla} \psi=\left(\partial_{\theta}+\frac{N-1}{2 \theta}\right) \gamma^{N} \psi+\frac{1}{\theta} \widetilde{\nabla}_{N-1} \psi,
$$

where $\widetilde{\nabla}_{N-1}$ is the Dirac operator on $\mathbb{S}^{N-1}$. The eigenfunctions of this operator 31,

$$
\widetilde{\nabla}_{N-1} \xi_{l m}^{( \pm)}= \pm i(l+(N-1) / 2) \xi_{l m}^{( \pm)}, \quad l=0,1, \ldots, m=0,1, \ldots, d_{l},
$$

may be used to find the spectral decomposition of Dirac operator on $\mathbb{R}^{N}$. We first set

$$
\hat{\xi}_{l m}^{( \pm)}=\frac{1}{\sqrt{2}}\left(\xi_{l m}^{(-)} \pm i \gamma^{N} \xi_{l m}^{(+)}\right) .
$$

In order to find the eigenfunctions of $\widetilde{\not}$ we insert the ansatz

$$
\chi_{k l m \pm}(\theta, \Omega)=c(k l)\left(a_{k l}(\theta) \hat{\xi}_{l m}^{(+)}(\Omega) \pm i b_{k l}(\theta) \hat{\xi}_{l m}^{(-)}(\Omega)\right), \quad \Omega \in \mathbb{S}^{N-1}
$$


into

$$
\widetilde{\nabla} \widetilde{\nabla} \chi_{k l m s}=-k^{2} \chi_{k l m s} .
$$

This leads to the differential equation

$$
\left[\partial_{\theta}^{2}+\frac{N-1}{\theta} \partial_{\theta}+\frac{l(l+N-2)}{\theta^{2}}+k^{2}\right] a_{k l}(\theta)=0,
$$

for $a_{k l}$ (and similarly $b_{k l}$ ). The unique regular solutions to these equations are given by Bessel functions,

$$
\begin{aligned}
& a_{k l}(\theta)=\theta^{-(N-2) / 2} J_{l+(N-2) / 2}(k \theta) \\
& b_{k l}(\theta)=\theta^{-(N-2) / 2} J_{l+N / 2}(k \theta) .
\end{aligned}
$$

The normalisation factor in Eq. (36) is determined from the condition

$$
\left\langle\chi_{k l m s}, \chi_{k^{\prime} l^{\prime} m^{\prime} s^{\prime}}\right\rangle=\delta\left(k-k^{\prime}\right) \delta_{l l^{\prime}} \delta_{m m^{\prime}} \delta_{s s^{\prime}},
$$

one finds $c(k l)=\sqrt{k / 2}$. In this work we also need the spectral function (Plancherel measure) defined by

$$
P_{N}(k)=\sum_{l m} \chi_{k l m s}(0)^{\dagger} \chi_{k l m s}^{(s)}(0) .
$$

From the expression Eq. (37) and behaviour of Bessel functions at $\theta=0$ it is seen that only the term with $l=0$ will contribute, leading to the result

$$
P_{N}(k)=\frac{k^{N-1}}{2^{N / 2} \operatorname{vol}\left(\mathbb{S}^{N-1}\right) \Gamma(N / 2)^{2}} .
$$

\section{VIII.2 Notions and results from microlocal analysis}

For convenience we mention some results and definitions from the theory of distributions and the theory of pseudodifferential operators (PDO's). If not indicated otherwise, these may be found in standard textbooks, for example [30, 13. PDO's generalise ordinary differential operators in the sense that they give meaning to fractional powers of derivatives. They are defined in terms of so-called symbols. We shall not give the most general definition of a symbol here, since only a certain class of symbols is important for this work.

Definition VIII.1. Let $\mathscr{O}$ be a subset of $\mathbb{R}^{n}$ and $m$ be a real number. Then a symbol of order $m$ is a function $a \in C^{\infty}\left(\mathscr{O}, \mathbb{R}^{n}\right)$ such that for every compact subset $K$ of $\mathscr{O}$ the following estimate holds

$$
\left|D_{x}^{\alpha} D_{\xi}^{\beta} a(x, \xi)\right| \leq C_{\alpha, \beta, K}(1+|\xi|)^{m-|\beta|}
$$

for all multiindices $\alpha, \beta$. $D^{\alpha}$ is $i^{|\alpha|} \partial_{1}^{\alpha_{1}} \ldots \partial_{n}^{\alpha_{n}}$. The set of all such symbols is denoted by $S^{m}\left(\mathscr{O}, \mathbb{R}^{n}\right)$ and one also writes $\mathrm{S}^{-\infty}=\bigcap_{m} \mathrm{~S}^{m}$.

There is the notion of the asymptotic expansion of a symbol which is an important tool for constructing PDO's. Suppose $a_{j} \in \mathrm{S}^{m_{j}}\left(\mathscr{O}, \mathbb{R}^{n}\right)$ for $j=$ 
$0,1,2, \ldots$ with $m_{j}$ monotonously decreasing to minus infinity. Then there exists $a \in \mathrm{S}^{m_{0}}\left(\mathscr{O}, \mathbb{R}^{n}\right)$ such that for all $N$

$$
a-\sum_{j=0}^{N} a_{j} \in \mathrm{S}^{m_{N}}\left(\mathscr{O}, \mathbb{R}^{n}\right)
$$

and $a$ is defined modulo $\mathrm{S}^{-\infty}$. One writes $a \sim \sum_{j} a_{j}$. If $a \in \mathrm{S}^{m}\left(\mathscr{O}, \mathbb{R}^{n}\right)$ then the operator

$$
A u(x)=\int e^{i x \xi} a(x, \xi) \hat{u}(\xi) \frac{d^{n} \xi}{(2 \pi)^{n}}
$$

is said to belong to $\mathrm{OP}^{m}(\mathscr{O})$, the PDO's of order $m$. $A$ is a continuous linear operator from $\mathscr{D}(\mathscr{O})$ to $C^{\infty}\left(\mathbb{R}^{n}\right)$. By the Schwartz kernel theorem it is thus given by a distribution kernel $K_{A} \in \mathscr{D}^{\prime}(\mathscr{O} \times \mathscr{O}) . K_{A}$ is smooth off the diagonal in $\mathscr{O} \times \mathscr{O}$ and smooth everywhere in $\mathscr{O} \times \mathscr{O}$ if $A \in \mathrm{OP}^{-\infty}(\mathscr{O})$. Hence the asymptotic expansion of a symbol uniquely determines a PDO modulo smoothing operators. The above statement carries over to matrix valued symbols without major changes. A principal symbol $\sigma_{m}(A)$ of $A \in \mathrm{OP}^{m}(\mathscr{O})$ is a representer of its symbol in $\mathrm{S}^{m}(\mathscr{O}) / \mathrm{S}^{m-1}(\mathscr{O})$. It can be chosen such that it transforms contravariantly under a change of coordinates (giving thus a well-defined function on the cotangent bundle) and it behaves multiplicatively under multiplication of two PDO's. On a manifold $M$ (or more generally on a vector-bundle $E$ ) PDO's are defined to be the continuous operators on $\mathscr{D}(M, E)$ which have the above properties in each coordinte patch.

We come to the definition of the polarisation set of a vector-valued distribution $u=\left(u^{1}, \cdots, u^{k}\right) \in \mathscr{D}^{\prime}(\mathscr{O})^{k}, \mathscr{O}$ an open subset of $\mathbb{R}^{n}$. For details of the definition and the subsequent results see $[3]$.

Definition VIII.2. The "polarisation set" $\mathrm{WF}_{\text {pol }}(u)$ of a vector-valued distribution $u$ is defined as

$$
\mathrm{WF}_{p o l}(u)=\bigcap_{A \in \mathrm{OP}^{0},} \mathscr{N}_{A u \in C^{\infty}},
$$

where

$$
\mathscr{N}_{A}=\left\{(x, \xi, w) \in T^{*} \mathscr{O} \times \mathbb{C}^{k} \mid \sigma_{0}(A)(x, \xi) w=0\right\} .
$$

From the transformation properties of the principal symbol it is clear that the definition can be carried over to the case of distributions with values in a vectorbundle $E . \mathrm{WF}_{\text {pol }}(u)$ is then seen to be a linear subset of $\pi^{*} E, \pi: T^{*} M \rightarrow M$ being the canonical projection in the fibres of the cotangent bundle. The "wave front set" $\mathrm{WF}(u)$ of a distribution is obtained by taking all points $(x, \xi) \in T^{*} M$ such that the fibre over this point in $\mathrm{WF}_{\text {pol }}(u)$ is nontrivial. The microlocal properties of the bidistributions considered in this work are more conveniently described in terms of their primed polarisation set, $\mathrm{WF}_{\text {pol }}^{\prime}$, which is obtained from the usual one by reversing the sign of the covectors in the second slot (the primed wave front set is defined similarly).

There is an important theorem on the polarisation set of distributions $u$ satisfying $P u \in C^{\infty}$ for differential operators $P$ of real principal type, which 
goes under the name 'propagation of singularities' 3, 13. Such operators are defined as follows (in the following we discuss the simple case where $P$ acts in the bundle $E=M \times \mathbb{C}^{k}$, but all results can be generalised in the obvious way to nontrivial vector bundles):

Definition VIII.3. A $k \times k$ system $P$ of differential operators on a manifold $M$ with principal symbol $p_{0}(x, \xi)$ is said to be of real principal type at $(y, \eta)$ if there exists a $k \times k$ symbol $\tilde{p}_{0}(x, \xi)$ such that

$$
\tilde{p}_{0}(x, \xi) p_{0}(x, \xi)=q(x, \xi) 1_{k}
$$

in a neighbourhood of $(y, \eta)$, where $q(x, \xi)$ is scalar and of scalar real principal type, i.e., $\partial_{\xi} q(x, \xi) \neq 0$ for all $\xi \neq 0$.

One sets

$$
\mathcal{Q}_{P}=\left\{(x, \xi) \mid \operatorname{det} p_{0}(x, \xi)=0\right\} .
$$

If $f$ is a $C^{\infty}$ function on $\mathcal{Q}_{P}$ with values in $\mathbb{C}^{k}$, then one defines

$$
D_{P} f=X_{q} f+\frac{1}{2}\left\{\tilde{p}_{0}, p_{0}\right\} f+i \tilde{p}_{0} p^{s} f,
$$

$X_{q}$ being the Hamiltonian vector field of $q$,

$$
\begin{aligned}
X_{q} & =\partial_{x} q \partial_{\xi}-\partial_{\xi} q \partial_{x}, \quad\left\{\tilde{p}_{0}, p_{0}\right\}=\partial_{\xi} \tilde{p}_{0} \partial_{x} p_{0}-\partial_{x} \tilde{p}_{0} \partial_{\xi} p_{0} \\
p^{s} & =p_{1}+\frac{1}{2} \partial_{\xi} D_{x} p_{0}, \quad \sigma(P) \sim p_{0}+p_{1}+p_{2}+\ldots
\end{aligned}
$$

One can prove that $D_{P}$ is a partial connection along the Hamiltonian vector field restricted to $\mathcal{Q}_{P}$. Since there is some arbitrariness in the choice of the symbol $\tilde{p}$, the partial connection is not uniquely defined. One can however prove that the remaining arbitrariness is irrelevant in what follows.

Definition VIII.4. A Hamilton orbit of a system $P$ of real principal type is a line bundle $\mathscr{L}_{P} \subset \mathscr{N}_{P} \uparrow c$, (c is an integral curve of the Hamiltonian field on $\left.\mathcal{Q}_{P}, \dot{c}(t)=X_{q}(c(t))\right)$ which is spanned by a sections $f$ satisfying $D_{P} f=0$, i.e. $\mathscr{L}_{P}$ is parallel with respect to the partial connection.

Theorem VIII.1. Let $P$ be of real principal type and $u$ a vector-valued distribution. Suppose $(x, \xi) \notin \mathrm{WF}(P u)$. Then, over a neighbourhood of $(x, \xi)$ in $\mathcal{Q}_{P}$, $\mathrm{WF}_{\text {pol }}(u)$ is a union of Hamilton orbits of $P$.

Aknowledgements: I would like to thank K. Fredenhagen, C.J. Fewster, B.S. Kay and especially M. Radzikowski and W. Junker for helpful discussions and comments. I am also grateful to K. Kratzert for pointing out his work to me and for noticing an error in the proof of Thm. IV.1] in an earlier version of this paper.

\section{References}

[1] R. Brunetti and K. Fredenhagen: 'Microlocal analysis and interacting quantum field theories: Renormalisation on physical backgrounds', Commun. Math. Phys. 208, 623-661 (2000) 
[2] R. Camporesi and A. Higuchi: 'On the eigenfunctions of the Dirac operator on spheres and real hyperbolic spaces', J. Geom. Phys. 20 1-18 (1996)

[3] N. Dencker: 'On the propagation of Polarisation Sets for Systems of Real Principal Type', Journal of Functional Analysis 46, 351-372 (1982)

[4] J. Dimock: 'Dirac quantum fields on a manifold', Trans. Am. Math. Soc. 269, 133-147 (1982)

[5] C. J. Fewster: 'A general worldline quantum inequality', Class. Quant. Grav. 17, 1897-1911 (2000)

[6] F.G. Friedlaender: 'The Wave Equation on Curved Space-Time', Cambridge University Press, Cambridge 1975

[7] S. A. Fulling, F. J. Narcowich, R. M. Wald: 'Singularity structure of the two-point function in quantum field theory in curved spacetime, II', Ann. Phys. 136, 243-272 (1981)

[8] G. Gonnella, B.S. Kay: 'Can locally Hadamard quantum states have nonlocal singularities?' Class. Quant. Grav. 6, 1445 (1989)

[9] P. Günther: 'Huygens' principle and hyperbolic equations', Academic Press, New York 1988

[10] R. Haag: 'Local quantum physics: Fields, particles, algebras', SpringerVerlag, Berlin 1992

[11] R. Haag and D. Kastler: 'An Algebraic Approach to Quantum Field Theory', J. Math. Phys. 5, 848-861 (1964)

[12] R. Haag, H. Narnhofer and U. Stein: 'On Quantum Field Theory in Gravitational Background', Commun. Math. Phys. 94, 219-238 (1984)

[13] L. Hörmander: 'Fourier integral operators I', Acta Math. 127 79-183 (1971), J. J. Duistermaat and L. Hörmander: 'Fourier integral operators II', Acta Math. 128, 183-269 (1972)

[14] L. Hörmander: 'The Analysis of Linear Partial Differential Operators III', Springer Verlag, Berlin 1985

[15] W. Junker: 'Hadamard states, adiabatic vacua and the construction of physical states for scalar quantum fields on curved space-time', Rev. Math. Phys. 8, 1091-1159 (1996)

[16] W. Junker: Erratum, in preparation.

[17] S. Hollands: PhD thesis, University of York, in preparation.

[18] B. S. Kay and R. M. Wald: 'Theorems on the uniqueness and thermal properties of stationary, nonsingular, quasifree states on spacetimes with a bifurcate Killing horizon', Phys. Rep. 207, 49 (1991)

[19] M. Köhler: 'The stress-energy tensor of a locally supersymmetric quantum field on a curved space-time', PhD thesis, Hamburg 1995 
[20] K. Kratzert: 'Singularitätsstruktur der Zweipunktfunktion des freien Diracfeldes in einer global hyperbolischen Raumzeit', DESY-THESIS-1999020, Hamburg 1999

[21] K. Kratzert: 'Singularity structure of the twopoint function of a free Dirac field on a globally hyperbolic spacetime', math-ph/0003015 to be published in Annalen der Physik.

[22] C. Lüders and J. E. Roberts: 'Local quasiequivalence and adiabatic vacuum states', Commun. Math. Phys. 134, 29 (1990)

[23] W. Moretti: 'Proof of the symmetry of the off-diagonal heat kernel and Hadamard's expansion coefficients in general $C^{\infty}$ Riemannian manifolds', gr-qc/9902034

[24] A. Najmi, A. Ottewil: 'Quantum states and the Hadamard form II. Energy Minimisation for spin 1/2 Fields', Phys. Rev. D 30, 2573-2584 (1984)

[25] L. Parker: 'Quantized fields and particle creation in expanding universes I', Phys. Rev. 183, 1057-1083 (1969)

[26] R. T. Powers and E. Størmer: 'Free states of the canonical anticommutation relations', Commun. Math. Phys. 16, 1 (1970)

[27] M. J. Radzikowski: 'Micro-Local Approach to the Hadamard condition in QFT on Curved Space-Time', Commun. Math. Phys. 179, 529-553 (1996)

[28] M. J. Radzikowski: 'A local to global singularity theorem for quantum field theory in curved space', Commun. Math. Phys. 180, 1-22 (1996)

[29] M. J. Radzikowski: Unpublished notes.

[30] M. E. Taylor: 'Pseudodifferential Operators', Princeton University Press, Princeton, New Jersey 1981 and 'Partial Differential Equations II', Springer Verlag, Berlin 1996

[31] A. Trautman: 'Spin structures on hypersurfaces and the spectrum of the Dirac operator on spheres', in: Spinors Twistors, Clifford Algebras and Quantum Deformations, Kluwer Academic Publishers, Dodrecht 1993

[32] R. Verch: 'Local definiteness, primarity and quasiequivalence of quasifree Hadamard quantum states in curved spacetime', Commun. Math. Phys. $160,507-536(1994)$

[33] R. Verch: 'Scaling Analysis and Ultraviolet Behaviour of Quantum Field Theories in Curved Spacetimes', PhD thesis, Hamburg 1996

[34] R. M. Wald: 'Quantum Field Theory in Curved Spacetime and Black Hole Thermodynamics', The University of Chicago Press, Chicago 1994

[35] M. Wellmann: 'Adiabatic vacuum states for the Dirac field on a curved spacetime', hep-th/9802087 